\title{
A Curved Boundary Treatment for Discontinuous Galerkin Schemes Solving Time Dependent Problems
}

\author{
Xiangxiong Zhang \\ Department of Mathematics, Purdue University, \\ 150 N. University Street, West Lafayette, IN 47907-2067
}

\begin{abstract}
For problems defined in a two-dimensional domain $\Omega$ with boundary conditions specified on a curve $\Gamma$, we consider discontinuous Galerkin (DG) schemes with high order polynomial basis functions on a geometry fitting triangular mesh. It is well known that directly imposing the given boundary conditions on a piecewise segment approximation boundary $\Gamma_{h}$ will render any finite element method to be at most second order accurate. Unless the boundary conditions can be accurately transferred from $\Gamma$ to $\Gamma_{h}$, in general curvilinear element method should be used to obtain high order accuracy. We discuss a simple boundary treatment which can be implemented as a modified DG scheme defined on triangles adjacent to $\Gamma_{h}$. Even though integration along the curve is still necessary, integrals over any curved element are avoided. If the domain $\Omega$ is convex, or if $\Omega$ is nonconvex and the true solutions can be smoothly extended to the exterior of $\Omega$, the modified DG scheme is high order accurate. In these cases, numerical tests on first order and second order partial differential equations including hyperbolic systems and the scalar wave equation suggest that it is as accurate as the full curvilinear DG scheme.
\end{abstract}

Keywords: discontinuous Galerkin, curved boundary, local discontinuous Galerkin method, high order accuracy, conservation laws, wave equations

\section{Introduction}

Consider solving a two-dimensional time dependent problem defined on a curved domain $\Omega$ with boundary conditions specified on a curve $\Gamma \subseteq \partial \Omega$. Assume a geometry fitting triangular mesh is given, we focus on discontinuous Galerkin (DG) method with high order polynomial basis functions. For high order schemes defined on such a triangular mesh as illustrated in Figure 1, boundary conditions on $\Gamma_{h}$ as an approximation to $\Gamma$ must be carefully treated to obtain optimal convergence rate. For instance, given homogeneous Dirichlet

Email address: zhan1966@purdue.edu (Xiangxiong Zhang)

Preprint submitted to Elsevier

December 14, 2015

(C) 2015. This manuscript version is made available under the Elsevier user license http://www.elsevier.com/open-access/userlicense/1.0/ 


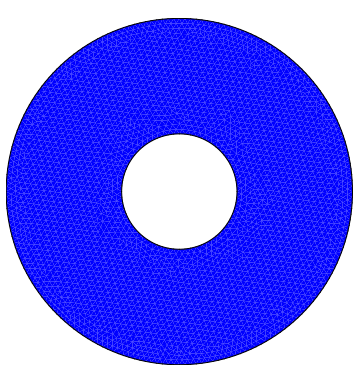

(a) The domain $\Omega$.

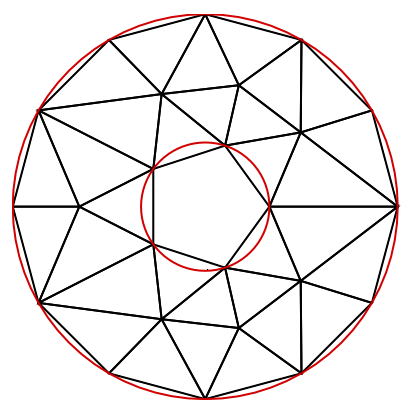

(b) $\Gamma$ consists of two red circles, approximated by piecewise segments $\Gamma_{h}$ in the triangular mesh.

Figure 1: An illustration of a geometry fitting triangular mesh on a curved domain.

boundary conditions on $\Gamma$, any finite element method will be at most second order accurate with Dirichlet boundary conditions imposed on $\Gamma_{h}[1,2]$. Towards optimal convergence rate, a curved element near $\Gamma$ can be used [3].

Even though the curvilinear element method via an isoparametric parametric approximation to $\Gamma[4]$ is rather convenient to use for DG schemes $[5,6,7,8]$, the computational and memory costs in curved elements will be increased due to integration on curved elements, especially when the boundary geometry is represented by very high order polynomials in high dimensions. Thus there is a strong motivation in studying more efficient alternatives to the full curvilinear DG methods.

One popular simple treatment to reduce computational cost of DG method on curved elements is to include the Jacobian determinant of the map from each curved element to a straight-sided reference element either in solution space or in test function space, e.g., [9, 10]. Even though it may work well for a lot of problems in practice, such a nonpolynomial approximation is not well understood in analysis. A low-storage curvilinear DG method was proposed and analyzed in [11, 12], where the geometric factors were included in both solution and test function spaces with a provable convergence under a mild condition on the mesh. For tensor-product type elements, the mass matrix is lumpable on the curved elements, see [8].

For specific schemes and problems, it is possible to accurately transfer the boundary conditions from $\Gamma$ to $\Gamma_{h}$ so that high order accuracy can be obtained for DG on triangular meshes without curved elements. In [13], a simple approximation to curved solid wall boundary conditions for steady gas dynamics equations was discussed. An implicit transfer of boundary conditions was presented in $[14,15]$ for the hybridizable DG method solving steady convection 
diffusion equations and the mesh does not need to be strictly geometry fitting in this method. For time dependent gas dynamics equations, an explicit transfer through an inverse Lax-Wendroff procedure was discussed for finite difference schemes in $[16,17,18,19]$ yet the performance of this method applied to DG schemes is unclear.

Recently a simple curved interface treatment for DG scheme on triangles solving acoustic wave equations was presented in [20]. In this paper, we will extend the approach in [20] to treating curved boundaries. We derive a modification to the DG scheme defined on a boundary triangle. Even though the line integration along the curve $\Gamma$ is still necessary, integrals over curved elements are avoided. By local truncation error analysis, such a modified DG scheme is high order accurate in convex domains. For nonconvex domains, it is also high order accurate if the equation and its smooth exact solution can be smoothly extended to the exterior of the domain. When the solution cannot be smoothly extended on nonconvex domains, the modified DG scheme is at most second order accurate however produces smaller errors than the DG scheme defined on triangles. On the other hand, a simple spectrum analysis suggest that this kind of modified DG scheme is unstable for arbitrary misfit between the boundary of a triangular mesh and the true curved boundary. Nonetheless, numerical tests suggest that such a scheme is stable on a reasonably coarse triangular mesh and finer ones.

The paper is organized as follows: we first discuss the main idea in Section 2 for first order equations. The same idea can be applied to other time dependent problems. As a demonstration, we discuss the second order wave equation in Section 3. For hyperbolic conservation laws, the local conservation is an important property. We discuss an additional step to enforce the local conservation in Section 4. Numerical tests are shown in Section 5. Section 6 consists of concluding remarks.

\section{Time-Dependent Conservation Laws}

\subsection{Preliminaries}

Consider solving the following initial-boundary value problem on a twodimensional curved domain $\Omega$ with $\Gamma \subseteq \partial \Omega$ :

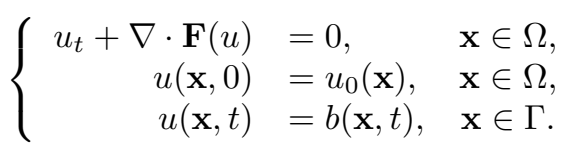

Suppose a triangular mesh $T_{h}$ of the domain $\Omega$ fitting the boundary $\partial \Omega$ is given. For simplicity, we assume that the mesh fits the geometry in a way that $\Gamma$ does not intersect any edge in $T_{h}$ at more than two points. We also assume $\Gamma$ does not pass more than two vertices of any triangle in $T_{h}$. These two assumptions are not essential for remaining discussion in this paper. Then for any boundary triangle $K$ adjacent to the curve $\Gamma$, there are only two possibilities for the intersection between $\Gamma$ and $K$. If $\Gamma$ intersects $K$ at only two vertices of 
$K$, we call it a convex case. Otherwise, $\Gamma$ also intersects $K$ at its interior, then we call it a concave case. Let $e_{K}^{i}(i=1,2,3)$ be the three edges of the triangle $K$ and $e_{k}^{1}$ be the one adjacent to the curve $\Gamma$. Let $\widetilde{e}_{K}^{1}$ be an isoparametric approximated representation of $\Gamma$, i.e., a high order polynomial interpolant of the curve $\Gamma$. We use $\widetilde{K}$ denote the curvilinear element bounded by $e_{K}^{2}, e_{K}^{3}$ and $\widetilde{e}_{K}^{1}$. Let $C$ denote the difference between $K$ and $\widetilde{K}$. See Figure 2 .

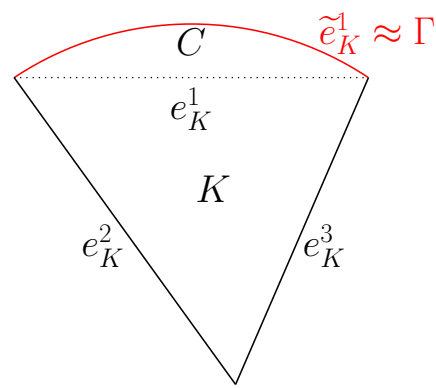

(a) Convex Case.

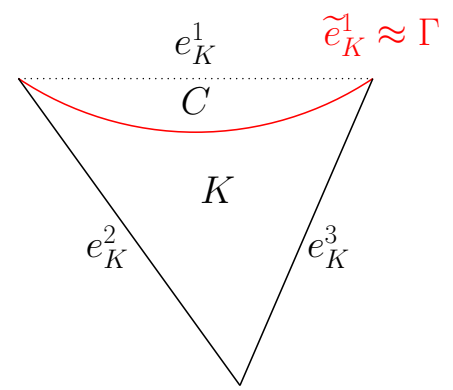

(b) Concave Case.

Figure 2: An illustration of a boundary triangle $K$ and the curvilinear element $\widetilde{K}$ bounded by the isoparametric curve $\widetilde{e}_{K}^{1}$ and two edges $e_{K}^{2}$ and $e_{K}^{3}$. The area bounded by $\widetilde{e}_{K}^{1}$ and the edge $e_{K}^{1}$ is denoted by $C$.

The following integration by parts formula will be used for a vector field $\mathbf{F}$ and a scalar function $v$,

$$
\iint_{\widetilde{K}} \mathbf{F} \cdot \nabla v d \mathbf{x}+\iint_{\widetilde{K}} \nabla \cdot \mathbf{F} v d \mathbf{x}=\int_{\partial \widetilde{K}} \mathbf{F} \cdot \mathbf{n}(\widetilde{K}) v
$$

where $\mathbf{n}(\widetilde{K})$ is the outward unit normal vector to the boundary $\partial \widetilde{K}$. Without further specification, $\mathbf{n}$ always denotes $\mathbf{n}(\widetilde{K})$ in the rest of this paper.

We recall the derivation of DG scheme on a curved element $\widetilde{K}$. Multiplying equation in (1) by a smooth test function $v$ on the element $\widetilde{K}$ and taking an integral, after integration by parts, we obtain

$$
\iint_{\widetilde{K}} u_{t} v d \mathbf{x}-\iint_{\widetilde{K}} \mathbf{F}(u) \cdot \nabla v d \mathbf{x}+\int_{\partial \widetilde{K}} \mathbf{F}(u) \cdot \mathbf{n} v d s=0 .
$$

In the weak formulation of a semi-discrete DG scheme, the solution $u_{h}$ defined on $\widetilde{K}$ satisfies the following equation for any polynomial test function $v_{h}$ :

$$
\iint_{\widetilde{K}} \frac{\partial}{\partial t} u_{h} v_{h} d \mathbf{x}-\iint_{\widetilde{K}} \mathbf{F}\left(u_{h}\right) \cdot \nabla v_{h} d \mathbf{x}+\int_{\partial \widetilde{K}} \widehat{\mathbf{F} \cdot \mathbf{n}} v_{h} d s=0,
$$

where $\widehat{\mathbf{F} \cdot \mathbf{n}}$ is a consistent numerical flux. Applying integration by parts on (2), we obtain the equivalent strong formulation:

$$
\iint_{\widetilde{K}}\left(\frac{\partial}{\partial t} u_{h}+\nabla \cdot \mathbf{F}\left(u_{h}\right)\right) v_{h} d \mathbf{x}+\int_{\partial \widetilde{K}}\left(\widehat{\mathbf{F} \cdot \mathbf{n}}-\mathbf{F}\left(u_{h}\right) \cdot \mathbf{n}\right) v_{h} d s=0,
$$




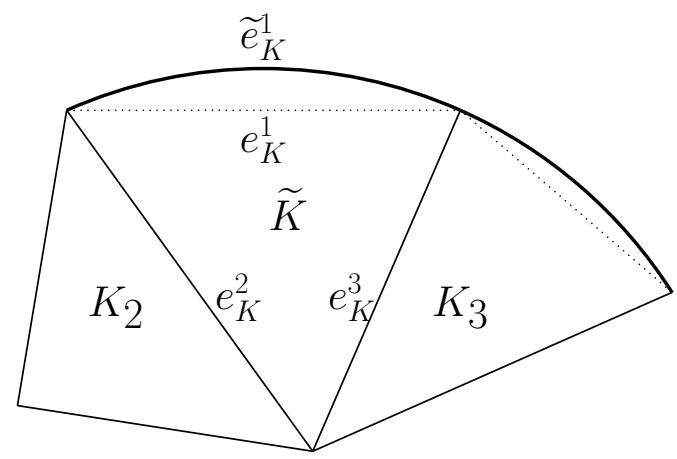

Figure 3: An illustration of neighbour cells.

There are quite a few popular numerical fluxes for nonlinear conservation laws (1), e.g., Godunov flux, Lax-Friedrichs flux, Roe flux and HLLE flux. Even though numerical performance of different fluxes may differ for specific problems, the choice of numerical fluxes does not affect the discussion in this paper. For simplicity, here we use the simplest Lax-Friedrichs flux with a global wave speed, which will be defined below. Let $K_{i}(i=2,3)$ be the triangle or the curved element which is adjacent to $\widetilde{K}$ and contains the edge $e_{K}^{i}$, see Figure 3. Let $u_{h}^{i}$ be the DG solution on $K_{i}(i=2,3)$. Let $\alpha$ be the maximum wave speed for the equation in (1), then the global Lax-Friedrichs flux in (2) is given by

$$
\left.\widehat{\mathbf{F} \cdot \mathbf{n}}\right|_{e_{K}^{i}}=\frac{1}{2}\left[\left.\mathbf{F}\left(u_{h}\right) \cdot \mathbf{n}\right|_{e_{K}^{i}}+\left.\mathbf{F}\left(u_{h}^{i}\right) \cdot \mathbf{n}\right|_{e_{K}^{i}}-\alpha\left(u_{h}^{i}-u_{h}\right)\right], \quad i=2,3,
$$

and

$$
\left.\widehat{\mathbf{F} \cdot \mathbf{n}}\right|_{\widetilde{e}_{K}^{1}}=\frac{1}{2}\left[\left.\mathbf{F}\left(u_{h}\right) \cdot \mathbf{n}\right|_{\widetilde{e}_{K}^{1}}+\left.\mathbf{F}(b) \cdot \mathbf{n}\right|_{\widetilde{e}_{K}^{1}}-\alpha\left(b-u_{h}\right)\right],
$$

where $b(x, y, t)$ is the boundary condition in (1).

\subsection{A Modified DG Scheme in the Convex Case}

We first discuss the convex case as shown in Figure 2 (a), where $\widetilde{K}=K \cup C$. By separating integrals over $K$ and $C$, the weak formulation of curved element DG scheme (2) is equivalent to

$$
\iint_{K} \frac{\partial}{\partial t} u_{h} v_{h} d \mathbf{x}-\iint_{K} \mathbf{F}\left(u_{h}\right) \cdot \nabla v_{h} d \mathbf{x}+\int_{\partial \widetilde{K}} \widehat{\mathbf{F} \cdot \mathbf{n}} v_{h} d s=\iint_{C}\left(-\frac{\partial}{\partial t} u_{h} v_{h}+\mathbf{F}\left(u_{h}\right) \cdot \nabla v_{h}\right) d \mathbf{x} .
$$

When $u_{h}$ is the exact smooth solution, it satisfies the equation in (1) thus

$$
\frac{\partial}{\partial t} u_{h}=-\nabla \cdot \mathbf{F}\left(u_{h}\right)
$$


Plugging (5) in, after integration by parts, the right hand side of (4) becomes

$$
\begin{aligned}
& \iint_{C}\left(-\frac{\partial}{\partial t} u_{h} v_{h}+\mathbf{F}\left(u_{h}\right) \cdot \nabla v_{h}\right) d \mathbf{x} \\
= & \iint_{C}\left(\nabla \cdot \mathbf{F}\left(u_{h}\right) v_{h}+\mathbf{F}\left(u_{h}\right) \cdot \nabla v_{h}\right) d \mathbf{x}=\int_{\partial C} \mathbf{F}\left(u_{h}\right) \cdot \mathbf{n}(C) v_{h} d s .
\end{aligned}
$$

where $\mathbf{n}(C)$ denotes the outward unit normal vector to the boundary $\partial C$. Replacing the right hand side in (4) by (6), we obtain a modified DG scheme in the convex case for a boundary triangle $K$ :

$\iint_{K} \frac{\partial}{\partial t} u_{h} v_{h} d \mathbf{x}-\iint_{K} \mathbf{F}\left(u_{h}\right) \cdot \nabla v_{h} d \mathbf{x}+\int_{\partial \widetilde{K}} \widehat{\mathbf{F} \cdot \mathbf{n}} v_{h} d s=\int_{\partial C} \mathbf{F}\left(u_{h}\right) \cdot \mathbf{n}(C) v_{h} d s$.

Applying integration by parts to $(7)$ and using the relation $\left.\mathbf{n}(C)\right|_{\widetilde{e}_{K}^{1}}=\left.\mathbf{n}\right|_{\widetilde{e}_{K}^{1}}$, after the cancellation of the line integral along the edge $e_{K}^{1}$, the strong formulation of the modified DG scheme can be written as

$$
\iint_{K}\left(\frac{\partial}{\partial t} u_{h}+\nabla \cdot \mathbf{F}\left(u_{h}\right)\right) v_{h} d \mathbf{x}+\int_{\partial \widetilde{K}}\left(\widehat{\mathbf{F} \cdot \mathbf{n}}-\mathbf{F}\left(u_{h}\right) \cdot \mathbf{n}\right) v_{h} d s=0 .
$$

Remark 1. A more straightforward way to derive (8) is to rewrite (3) as

$$
\begin{aligned}
& \iint_{K}\left(\frac{\partial}{\partial t} u_{h}+\nabla \cdot \mathbf{F}\left(u_{h}\right)\right) v_{h} d \mathbf{x}+\int_{\partial \widetilde{K}}\left(\widehat{\mathbf{F} \cdot \mathbf{n}}-\mathbf{F}\left(u_{h}\right) \cdot \mathbf{n}\right) v_{h} d s \\
= & -\iint_{C}\left(\frac{\partial}{\partial t} u_{h}+\nabla \cdot \mathbf{F}\left(u_{h}\right)\right) v_{h} d \mathbf{x} .
\end{aligned}
$$

Then plug (5) into the right hand side.

Remark 2 (Local Truncation Error). The only difference between (3) and (8) is $\iint_{C}\left(\frac{\partial}{\partial t} u_{h}+\nabla \cdot \mathbf{F}\left(u_{h}\right)\right) v_{h} d \mathbf{x}$, which is equal to zero if $u_{h}$ is equal to an exact smooth solution $u$. If we define the local truncation error of a semi-discrete scheme as the residue after substituting $u_{h}$ by the exact solution in the scheme, then the local truncation error of the modified scheme (7) and (8) is the same as the curvilinear DG method (2) and (3).

Remark 3 (Stability). A simple spectrum analysis in Appendix B suggests that the distance between the mesh boundary and the true boundary must be small enough for the sake of stability. This means that the modified DG scheme is not stable on a very coarse triangular mesh. On the other hand, the stability of such a modified scheme is highly nontrivial to establish even if assuming the area $C$ is very small with respect to $K$ in Figure 2. See Appendix A for a discussion of a one-dimensional modified scheme. Nonetheless, numerical tests suggest the modified DG scheme is stable on reasonably coarse meshes and their refined meshes in examples considered in Section 5. 


\subsection{The Concave Case}

For the concave case as shown in Figure 2 (b), we have $K=\widetilde{K} \cup C$. Adding integrals on $C$ to both sides, (2) is equivalent to

$\iint_{K} \frac{\partial}{\partial t} u_{h} v_{h} d \mathbf{x}-\iint_{K} \mathbf{F}\left(u_{h}\right) \cdot \nabla v_{h} d \mathbf{x}+\int_{\partial \widetilde{K}} \widehat{\mathbf{F} \cdot \mathbf{n}} v_{h} d s=\iint_{C}\left(\frac{\partial}{\partial t} u_{h} v_{h}-\mathbf{F}\left(u_{h}\right) \cdot \nabla v_{h}\right) d \mathbf{x}$.

If the partial differential equation and its solution can be smoothly extended to $C$, assume $u_{h}$ is equal to this smooth extension, then (5) is satisfied on $C$. Plugging (5) in, after integration by parts, the right hand side of (9) becomes

$$
\begin{aligned}
& \iint_{C}\left(\frac{\partial}{\partial t} u_{h} v_{h}-\mathbf{F}\left(u_{h}\right) \cdot \nabla v_{h}\right) d \mathbf{x} \\
= & -\iint_{C}\left(\nabla \cdot \mathbf{F}\left(u_{h}\right) v_{h}+\mathbf{F}\left(u_{h}\right) \cdot \nabla v_{h}\right) d \mathbf{x}=-\int_{\partial C} \mathbf{F}\left(u_{h}\right) \cdot \mathbf{n}(C) v_{h} d s(10)
\end{aligned}
$$

where $\mathbf{n}(C)$ denotes the outward unit normal vector to the boundary $\partial C$. Replacing the right hand side in (9) by (10), we obtain a modified DG scheme in the concave case for a boundary triangle $K$ :

$\iint_{K} \frac{\partial}{\partial t} u_{h} v_{h} d \mathbf{x}-\iint_{K} \mathbf{F}\left(u_{h}\right) \cdot \nabla v_{h} d \mathbf{x}+\int_{\partial \widetilde{K}} \widehat{\mathbf{F} \cdot \mathbf{n}} v_{h} d s=-\int_{\partial C} \mathbf{F}\left(u_{h}\right) \cdot \mathbf{n}(C) v_{h} d s$,

Applying integration by parts to (11) and using the relation $\left.\mathbf{n}(C)\right|_{\tilde{e}_{K}^{1}}=-\left.\mathbf{n}\right|_{\tilde{e}_{K}^{1}}$, the strong formulation of the modified DG scheme in the concave case is exactly the same as in the convex case (8).

Remark 4 (Local Truncation Error). If the partial differential equation and its solution can be smoothly extended to $C$, then discussion of the local truncation error in the concave case is the same as in Remark 2, i.e., the local truncation error of the modified DG scheme is as small as the curvilinear DG scheme. Otherwise, the modified DG scheme cannot be high order accurate. Let $u$ be a function defined on $K$ denoting the extension of the exact solution. Assume there is a singularity in $u$ along $\Gamma$, then it is impossible to approximate the function $u$ to the optimal order in the triangle $K$ by polynomials. Thus any integral term over $K$ will destroy high order accuracy in DG scheme with high order polynomial basis functions.

\subsection{A Simple Implementation}

Compared to the curved element method (3), we can see that integrals over $\widetilde{K}$ are avoided in the modified DG scheme (8), even though integration along $\partial \widetilde{K}$ is still needed. The modified DG scheme can be implemented as a simple modification to the DG scheme defined on the triangle $K$. 
The strong formulation of the modified DG scheme can be written as

$$
\begin{aligned}
& \iint_{K}\left(\frac{\partial}{\partial t} u_{h}+\nabla \cdot \mathbf{F}\left(u_{h}\right)\right) v_{h} d \mathbf{x}+\sum_{i=2}^{3} \int_{e_{K}^{i}}\left(\widehat{\mathbf{F} \cdot \mathbf{n}}-\mathbf{F}\left(u_{h}\right) \cdot \mathbf{n}\right) v_{h} d s \\
+ & \int_{\widetilde{e}_{K}^{1}}\left(\widehat{\mathbf{F} \cdot \mathbf{n}}-\mathbf{F}\left(u_{h}\right) \cdot \mathbf{n}\right) v_{h} d s=0 .
\end{aligned}
$$

Since $K$ and $\widetilde{K}$ share two edges, the strong formulation of DG scheme defined on the triangle $K$ is

$$
\begin{aligned}
& \iint_{K}\left(\frac{\partial}{\partial t} u_{h}+\nabla \cdot \mathbf{F}\left(u_{h}\right)\right) v_{h} d \mathbf{x}+\sum_{i=2}^{3} \int_{e_{K}^{i}}\left(\widehat{\mathbf{F} \cdot \mathbf{n}}-\mathbf{F}\left(u_{h}\right) \cdot \mathbf{n}\right) v_{h} d s \\
+ & \left.\int_{e_{K}^{1}}(\widehat{\mathbf{F} \cdot \mathbf{n}(K})-\mathbf{F}\left(u_{h}\right) \cdot \mathbf{n}(K)\right) v_{h} d s=0 .
\end{aligned}
$$

where $\mathbf{n}(K)$ denotes the outward unit normal vector to the boundary of the triangle $K$.

Therefore, the modified scheme (8) can be implemented as a simple modification to DG scheme defined on triangles (12) as follows: on each triangle $K$ adjacent to the curve $\Gamma$ as shown in Figure 2, in (12),

REPLACE $\left.\int_{e_{K}^{1}}(\widehat{\mathbf{F} \cdot \mathbf{n}(K})-\mathbf{F}\left(u_{h}\right) \cdot \mathbf{n}(K)\right) v_{h} d s \quad \mathrm{BY} \int_{\widetilde{e}_{K}^{1}}\left(\widehat{\mathbf{F} \cdot \mathbf{n}}-\mathbf{F}\left(u_{h}\right) \cdot \mathbf{n}\right) v_{h} d s$,

where in the convex case, for $u_{h}$ defined on $K$, the extension of $u_{h}$ to $\widetilde{e}_{K}^{1}$ is simply the evaluation of the DG polynomial on $K$ at $\widetilde{e}_{K}^{1}$.

Remark 5. As discussed in Remark 4, the modification (13) does not improve the order of convergence for the DG scheme on triangles (12) for a problem on a nonconvex domain with no smooth extensions of the exact solution. For example, the extension of the exact solution is not smooth across the solid wall boundary in modeling flow passing a cylinder. However, the modification (13) does reduce errors in the last line integral in (12). For example, consider imposing homogeneous Dirichlet boundary condition on $\Gamma$, i.e., $b(x, y, t) \equiv 0$ in (1). Suppose $\Gamma$ is an inflow boundary and we use upwind flux $\mathbf{F \cdot \mathbf { n } ( K )}=$ $\mathbf{F}(0) \cdot \mathbf{n}(K)$ on $e_{K}^{1}$ in (12). Then the Dirichlet boundary condition is enforced on $e_{K}^{1}$ and $\int_{e_{K}^{1}}\left(\mathbf{F}(0) \cdot \mathbf{n}(K)-\mathbf{F}\left(u_{h}\right) \cdot \mathbf{n}(K)\right) v_{h} d s$ induces at least a second order error. On the other hand, if $u_{h}=u$ with $u$ being the exact smooth solution, then $\int_{\widetilde{e}_{K}^{1}}\left(\mathbf{F}(0) \cdot \mathbf{n}-\mathbf{F}\left(u_{h}\right) \cdot \mathbf{n}\right) v_{h} d s$ is a high order error term in the local truncation error.

Remark 6. The modified DG scheme can also be applied to a curved material interface problem [20]. Consider a triangle of the concave case as shown in Figure 2 (b) where the red curve denotes the interface for the curved interface problem, the exact solution is not smooth across the curve thus the modified 
DG scheme is not high order accurate. Nonetheless, the modified DG scheme does produce smaller errors than the DG scheme defined on triangles, see an accuracy test in [20].

\section{The Second Order Wave Equation}

In the previous section, we have derived a modified DG scheme (8) for first order equations by using the partial differential equation (5), i.e., replacing the time derivative by the spatial derivatives. As mentioned in Remark (1), it is also equivalent to removing the volume integral over $C$ illustrated in Figure 2 in the standard DG scheme. This simple idea can be easily applied to DG schemes solving equations with higher order derivatives such as convection diffusion equations. As a demonstration, we discuss the local DG (LDG) method $[21,22]$ solving the scalar wave equation:

$$
\left\{\begin{aligned}
u_{t t}-\nabla \cdot\left(c(\mathbf{x})^{2} \nabla u\right) & =0, & & \mathbf{x} \in \Omega \\
u(\mathbf{x}, 0) & =u_{0}(\mathbf{x}), & & \mathbf{x} \in \Omega \\
u_{t}(\mathbf{x}, 0) & =v_{0}(\mathbf{x}), & & \mathbf{x} \in \Omega \\
u(\mathbf{x}, t) & =b(\mathbf{x}, t), & & \mathbf{x} \in \Gamma
\end{aligned}\right.
$$

All notations for meshes are the same as in the previous section.

\subsection{The $L D G$ Method}

Let $\mathbf{q}=\left(q^{1}, q^{2}\right)$ denote an auxiliary variable. Let $\mathbf{F}=(u, 0)$ and $\mathbf{G}=(0, u)$. Then the wave equation can be written as a first order system,

$$
\left\{\begin{array}{rl}
u_{t t} & =\nabla \cdot(c \mathbf{q}) \\
q^{1} & =c \nabla \cdot \mathbf{F} \\
q^{2} & =c \nabla \cdot \mathbf{G}
\end{array} .\right.
$$

Multiplying (15) by test functions $\psi, \phi$ and $\varphi$ on the element $\widetilde{K}$ and taking an integral, after integration by parts, we obtain

$$
\begin{aligned}
\iint_{\widetilde{K}} u_{t t} \psi d \mathbf{x} & =-\iint_{\widetilde{K}} c \mathbf{q} \cdot \nabla \psi d \mathbf{x}+\int_{\partial \widetilde{K}} c \mathbf{q} \cdot \mathbf{n} \psi d s \\
\iint_{\widetilde{K}} q^{1} \phi d \mathbf{x} & =-\iint_{\widetilde{K}} \mathbf{F} \cdot \nabla(c \phi) d \mathbf{x}+\int_{\partial \widetilde{K}} c \mathbf{F} \cdot \mathbf{n} \phi d s, \\
\iint_{\widetilde{K}} q^{2} \varphi d \mathbf{x} & =-\iint_{\widetilde{K}} \mathbf{G} \cdot \nabla(c \varphi) d \mathbf{x}+\int_{\partial \widetilde{K}} c \mathbf{G} \cdot \mathbf{n} \varphi d s .
\end{aligned}
$$

The LDG method for (15) on a curved element $\widetilde{K}$ is formulated as follows:

$$
\begin{aligned}
\iint_{\widetilde{K}} \frac{\partial^{2}}{\partial t^{2}} u_{h} \psi_{h} d \mathbf{x} & =-\iint_{\widetilde{K}} c \mathbf{q}_{h} \cdot \nabla \psi_{h} d \mathbf{x}+\int_{\partial \widetilde{K}} \widehat{c \mathbf{q} \cdot \mathbf{n}} \psi_{h} d s \\
\iint_{\widetilde{K}} q_{h}^{1} \phi_{h} d \mathbf{x} & =-\iint_{\widetilde{K}} \mathbf{F}\left(u_{h}\right) \cdot \nabla\left(c \phi_{h}\right) d \mathbf{x}+\int_{\partial \widetilde{K}} \widehat{c \mathbf{F} \cdot \mathbf{n}} \phi_{h} d s \\
\iint_{\widetilde{K}} q_{h}^{2} \varphi_{h} d \mathbf{x} & =-\iint_{\widetilde{K}} \mathbf{G}\left(u_{h}\right) \cdot \nabla\left(c \varphi_{h}\right) d \mathbf{x}+\int_{\partial \widetilde{K}} \widehat{c \mathbf{G} \cdot \mathbf{n} \varphi_{h}} d s
\end{aligned}
$$


where $u_{h}$ and $\mathbf{q}_{h}$ are solutions and $\psi_{h}, \phi_{h}$ and $\varphi_{h}$ are test functions.

After integration by parts, we obtain the equivalent strong formulation of the LDG scheme,

$$
\begin{aligned}
\iint_{\widetilde{K}} \frac{\partial^{2}}{\partial t^{2}} u_{h} \psi_{h} d \mathbf{x} & =\iint_{\widetilde{K}} \nabla \cdot\left(c \mathbf{q}_{h}\right) \psi_{h} d \mathbf{x}+\int_{\partial \widetilde{K}}\left(\widehat{c \mathbf{q} \cdot \mathbf{n}}-c \mathbf{q}_{h} \cdot \mathbf{n}\right) \psi_{h} d s \\
\iint_{\widetilde{K}} q_{h}^{1} \phi_{h} d \mathbf{x} & =\iint_{\widetilde{K}} c \nabla \cdot \mathbf{F}\left(u_{h}\right) \phi_{h} d \mathbf{x}+\int_{\partial \widetilde{K}}\left(\widehat{c \mathbf{F} \cdot \mathbf{n}}-c \mathbf{F}\left(u_{h}\right) \cdot \mathbf{n}\right) \phi_{h} d s \\
\iint_{\widetilde{K}} q_{h}^{2} \varphi_{h} d \mathbf{x} & =\iint_{\widetilde{K}} c \nabla \cdot \mathbf{G}\left(u_{h}\right) \varphi_{h} d \mathbf{x}+\int_{\partial \widetilde{K}}\left(\widehat{c \mathbf{G} \cdot \mathbf{n}}-c \mathbf{G}\left(u_{h}\right) \cdot \mathbf{n}\right) \varphi_{h} d s
\end{aligned}
$$

The numerical fluxes can be taken in an alternating fashion, see [22].

\subsection{The Modified LDG Scheme}

Following Remark 1, we can rewrite the strong formulation of LDG scheme as

$$
\begin{gathered}
\iint_{K}\left(\left(u_{h}\right)_{t t}-\nabla \cdot\left(c \mathbf{q}_{h}\right)\right) \psi_{h} d \mathbf{x}-\int_{\partial \widetilde{K}}\left(\widehat{c \mathbf{q} \cdot \mathbf{n}}-c \mathbf{q}_{h} \cdot \mathbf{n}\right) \psi_{h} d s=\iint_{C}\left(\left(u_{h}\right)_{t t}-\nabla \cdot\left(c \mathbf{q}_{h}\right)\right) \psi_{h} d \mathbf{x}, \\
\iint_{K}\left(q_{h}^{1}-c \nabla \cdot \mathbf{F}\left(u_{h}\right)\right) \phi_{h} d \mathbf{x}-\int_{\partial \widetilde{K}}\left(\widehat{c \mathbf{F} \cdot \mathbf{n}}-c \mathbf{F}\left(u_{h}\right) \cdot \mathbf{n}\right) \phi_{h} d s=\iint_{C}\left(q_{h}^{1}-c \nabla \cdot \mathbf{F}\left(u_{h}\right)\right) \phi_{h} d \mathbf{x}, \\
\iint_{K}\left(q_{h}^{2}-c \nabla \cdot \mathbf{G}\left(u_{h}\right)\right) \varphi_{h} d \mathbf{x}-\int_{\partial \widetilde{K}}\left(\widehat{c \mathbf{G} \cdot \mathbf{n}}-c \mathbf{G}\left(u_{h}\right) \cdot \mathbf{n}\right) \varphi_{h} d s=\iint_{C}\left(q_{h}^{2}-c \nabla \cdot \mathbf{G}\left(u_{h}\right)\right) \varphi_{h} d \mathbf{x} .
\end{gathered}
$$

Set the right hand side as zero, then we obtain the strong formulation of modified LDG scheme,

$$
\begin{aligned}
\iint_{K} \frac{\partial^{2}}{\partial t^{2}} u_{h} \psi_{h} d \mathbf{x} & =\iint_{K} \nabla \cdot\left(c \mathbf{q}_{h}\right) \psi_{h} d \mathbf{x}+\int_{\partial \widetilde{K}}\left(\widehat{c \mathbf{q} \cdot \mathbf{n}}-c \mathbf{q}_{h} \cdot \mathbf{n}\right) \psi_{h} d s \\
\iint_{K} q_{h}^{1} \phi_{h} d \mathbf{x} & =\iint_{K} c \nabla \cdot \mathbf{F}\left(u_{h}\right) \phi_{h} d \mathbf{x}+\int_{\partial \widetilde{K}}\left(\widehat{c \mathbf{F} \cdot \mathbf{n}}-c \mathbf{F}\left(u_{h}\right) \cdot \mathbf{n}\right) \phi_{h} d s \\
\iint_{K} q_{h}^{2} \varphi_{h} d \mathbf{x} & =\iint_{K} c \nabla \cdot \mathbf{G}\left(u_{h}\right) \varphi_{h} d \mathbf{x}+\int_{\partial \widetilde{K}}\left(\widehat{c \mathbf{G} \cdot \mathbf{n}}-c \mathbf{G}\left(u_{h}\right) \cdot \mathbf{n}\right) \varphi_{h} d s
\end{aligned}
$$

The modified scheme above can be easily implemented as a simple modification to LDG scheme defined on a triangle $K$.

\section{Local Conservation for Conservation Laws}

Integrating $u_{t}+\nabla \cdot \mathbf{F}(u)=0$ on any cell $T$ in a triangulation, we obtain the integral form of conservation laws,

$$
\frac{d}{d t} \iint_{T} u d \mathbf{x}=-\int_{\partial T} \mathbf{F} \cdot \mathbf{n} d s
$$

The semi-discrete analog of (16) takes the form

$$
\frac{d}{d t} \iint_{T} u_{h} d \mathbf{x}=-\int_{\partial T} \widehat{\mathbf{F} \cdot \mathbf{n}} d s
$$


where $u_{h}$ is the numerical solution and $\widehat{\mathbf{F} \cdot \mathbf{n}}$ is the numerical flux. Conservative schemes are those satisfying (17). For nonlinear conservation laws, conservative schemes are preferred for several reasons. First, local conservation implies global conservation, which is a crucial property for stability. Second, Lax-Wendroff theorem states that the limit of solutions of conservative schemes will be a weak solution to the conservation law as mesh refines. Third, the location of shock waves computed by nonconservative schemes is usually wrong.

By taking the test function $v_{h} \equiv 1$ in (2), we can see that the curvilinear DG scheme satisfies the local conservation,

$$
\frac{d}{d t} \iint_{\widetilde{K}} u_{h} d \mathbf{x}=-\int_{\partial \widetilde{K}} \widehat{\mathbf{F} \cdot \mathbf{n}} d s .
$$

For the modified scheme, taking $v_{h} \equiv 1$ in (7), we obtain,

$$
\frac{d}{d t} \iint_{K} u_{h} d \mathbf{x}-\int_{\partial C} \mathbf{F}\left(u_{h}\right) \cdot \mathbf{n}(C) d s=-\int_{\partial \widetilde{K}} \widehat{\mathbf{F} \cdot \mathbf{n}} d s .
$$

Obviously the modified scheme is not conservative any more. On the other hand, assuming (5) holds, by the divergence theorem, we have

$$
-\int_{\partial C} \mathbf{F}\left(u_{h}\right) \cdot \mathbf{n}(C) d s=-\iint_{C} \nabla \cdot \mathbf{F}\left(u_{h}\right) d \mathbf{x}=\frac{d}{d t} \iint_{C} u_{h} d \mathbf{x} .
$$

Thus the modified DG scheme satisfies the local conservation (18) up to the error

$$
\iint_{C}\left(\frac{\partial}{\partial t} u_{h}+\nabla \cdot \mathbf{F}\left(u_{h}\right)\right) d \mathbf{x}
$$

which might be arbitrarily large for nonsmooth problems.

In this section, we discuss an additional step to enforce the local conservation in the modified scheme. For simplicity, we first discuss the Euler forward time discretization. Let $n$ denote the time step index and $|\widetilde{K}|$ denote the area of the cell $\widetilde{K}$, the desired property of local conservation (18) with Euler forward is

$$
\frac{1}{|\widetilde{K}|} \iint_{\widetilde{K}} u_{h}^{n+1} d \mathbf{x}=\frac{1}{|\widetilde{K}|} \iint_{\widetilde{K}} u_{h}^{n} d \mathbf{x}-\frac{\Delta t}{|\widetilde{K}|} \int_{\partial \widetilde{K}} \widehat{\mathbf{F} \cdot \mathbf{n}} d s,
$$

which means that the change of cell average of $u_{h}$ in time is only up to the numerical flux on the boundary of $\widetilde{K}$. Let $\bar{u}_{h}^{n}=\frac{1}{|\widetilde{K}|} \iint_{\widetilde{K}} u_{h}^{n} d \mathbf{x}$. After the DG polynomials $u_{h}^{n+1}$ are computed from the modified scheme, we need to adjust its cell average so that $\bar{u}_{h}^{n+1}$ satisfies (20).

To enforce (20), three quantities must be computed, i.e., $\bar{u}_{h}^{n}, \bar{u}_{h}^{n+1}$ and $\int_{\partial \widetilde{K}} \widehat{\mathbf{F} \cdot \mathbf{n}} d s$. Notice that the numerical flux $\int_{\partial \widetilde{K}} \widehat{\mathbf{F} \cdot \mathbf{n}} d s$ is included in the modified scheme (7). Even though $\bar{u}_{h}^{n}$ is only a degree of freedom of the DG polynomial, it is not explicitly available in the proposed modified scheme (7) because the motivation of introducing the modified scheme is to avoid integrals over $\widetilde{K}$. Let $\overline{\mathbf{x}}_{i}$ and $\bar{w}_{i}\left(i=1, \cdots, N_{c}\right)$ be the quadrature points and weights 
on $\widetilde{K}$ which are exact for computing $\iint_{\widetilde{K}} u_{h}^{n} d \mathbf{x}$. Such a quadrature rule can be obtained by considering a map from $\widetilde{K}$ to a reference triangle and sufficiently many quadrature points on the reference triangle, e.g, see [7].

Suppose $u_{h}^{n}(\mathbf{x})$ is a bivariate polynomial of degree $N$ on $K$. Let $\mathbf{u}_{h}^{n}$ denote the column vector consisting of nodal values on $\frac{(N+1)(N+2)}{2}$ nodes of the triangle $K$ such that $u_{h}^{n}(\mathbf{x})$ is uniquely determined by $\mathbf{u}_{h}^{n}$. In nodal DG method [7], $\mathbf{u}_{h}^{n}$ will be used in computation explicitly on each cell. To compute $u_{h}^{n}\left(\overline{\mathbf{x}}_{i}\right)$ based on $\mathbf{u}_{h}^{n}$, one can first convert the nodal representation of a polynomial to modal representation then evaluate the basis polynomials at $\overline{\mathbf{x}}_{i}$, see [7]. Such a process is a linear map from the vector $\mathbf{u}_{h}^{n}$ to $u_{h}^{n}\left(\overline{\mathbf{x}}_{i}\right)$. Let $A_{K}\left(\overline{\mathbf{x}}_{i}\right)$ be the matrix representation of this linear map, i.e., $u_{h}^{n}\left(\overline{\mathbf{x}}_{i}\right)=A_{K}\left(\overline{\mathbf{x}}_{i}\right) \mathbf{u}_{h}^{n}$. Notice that $A_{K}\left(\overline{\mathbf{x}}_{i}\right)$ is actually a row vector and it depends only on the triangle $K$ and $\overline{\mathbf{x}}_{i}$ for fixed $N$. Let $A_{K}$ denote the matrix $\left[A_{K}\left(\mathbf{x}_{1}\right)^{T}, \cdots, A_{K}\left(\mathbf{x}_{N_{c}}\right)^{T}\right]^{T}$ where $A^{T}$ means the transpose of the matrix $A$. Let $\bar{W}$ denote the row vector $\left[\bar{w}_{1}, \bar{w}_{2}, \cdots, \bar{w}_{N_{c}}\right]$. Then we have

$$
\iint_{\widetilde{K}} u_{h}^{n} d \mathbf{x}=\sum_{i=1}^{N_{c}} u_{h}^{n}\left(\overline{\mathbf{x}}_{i}\right) \bar{w}_{i}=\bar{W} A_{K} \mathbf{u}_{h}^{n}=\bar{A}_{\widetilde{K}} \mathbf{u}_{h}^{n},
$$

where $\bar{A}_{\widetilde{K}}=\bar{W} A_{K}$ is a vector of the same size as $\mathbf{u}_{h}^{n}$ and it depends only on polynomial degree $N$ and the cells $K$ and $\widetilde{K}$. In other words, the cell average $\bar{u}_{h}^{n}$ over $\widetilde{K}$ can be easily computed by a dot product of two vectors if we compute $\bar{A}_{\widetilde{K}}$ offline and store it.

We summarize the conservation correction step for Euler forward above as follows:

1. Given the DG polynomial $u_{h}^{n}(\mathbf{x})$ on time step $n$, evolve to next time step by the modified scheme (8) with Euler forward and store $\int_{\partial \widetilde{K}} \widehat{\mathbf{F} \cdot \mathbf{n}} d s$. Obtain the DG polynomial $u_{h}^{n+1}(\mathbf{x})$.

2. Compute two cell averages $\bar{u}_{h}^{n}$ and $\bar{u}_{h}^{n+1}$ by two vector dot products as in (21) with precomputed vector $\bar{A}_{\widetilde{K}}$.

3. The corrected DG polynomial is given by a simple translation

$$
\widetilde{u}_{h}^{n+1}(\mathbf{x})=u_{h}^{n+1}(\mathbf{x})-\bar{u}_{h}^{n+1}+\bar{u}_{h}^{n}-\frac{\Delta t}{|\widetilde{K}|} \int_{\partial \widetilde{K}} \widehat{\mathbf{F} \cdot \mathbf{n}} d s .
$$

It is straightforward to see that the corrected DG polynomial satisfies (20). For higher order time discretizations, the correction step above can be used for each time stage in Runge-Kutta methods or each time step in multi-step methods to enforce the conservation.

Remark 7. Compared to the modified scheme (8), the additional computational cost of the conservation correction step is marginal, which is due to the fact that the correct numerical flux integral $\int_{\partial \widetilde{K}} \widehat{\mathbf{F} \cdot \mathbf{n}} d s$ is used in the modified scheme (8). On the other hand, the storage cost is doubled since $\bar{A}_{\widetilde{K}}$ must be stored for each triangle adjacent to curved boundaries. 
Remark 8. For nonlinear conservation laws, linear high order schemes such as DG method is not stable when shocks emerge. Nonlinear limiters must be used to stabilize the DG scheme in practice, e.g., the minmod type TVB limiter [23], WENO type limiter $[24,25]$ and positivity-preserving limiter [26, 27, 28]. Such limiters are all defined for conservative schemes thus they do not apply to the nonconservative modified DG scheme (8). With the conservation correction, it is straightforward to extend these limiters to the modified DG scheme for solving nonlinear conservation laws.

\section{Numerical Tests}

In this section, we test the performance of the modified DG scheme in several examples. For the time discretization, we use the fourth-order low storage Runge-Kutta for conservation laws (1) and a fourth-order symplectic time integrator for the second order wave equation (14). See [7] and [22] for more implementation details. We use the upwind flux for linear equations and the Lax-Friedrichs flux with a global maximum of wave speed for nonlinear equations for their simplicity. Other types of fluxes may result in much better performance in specific context, e.g., Roe flux was used for nonlinear gas dynamics equations with solid wall boundary conditions in [13].

\subsection{Homogeneous Dirichlet Boundary Conditions}

\section{Example 1.}

Consider solving the two-dimensional Maxwell's equations in transverse magnetic form in a unit radius cylindrical cavity,

$$
\begin{aligned}
\frac{\partial H^{x}}{\partial t} & =-\frac{\partial E^{z}}{\partial y} \\
\frac{\partial H^{y}}{\partial t} & =\frac{\partial E^{z}}{\partial x} \\
\frac{\partial E^{z}}{\partial t} & =\frac{\partial H^{y}}{\partial x}-\frac{\partial H^{x}}{\partial y} .
\end{aligned}
$$

The computational domain is a unit disk with the boundary circle being perfect electrical conductor (PEC). Thus the boundary condition is $E^{z}=0$. An exact solution in polar coordinates $(r, \theta)$ is given by $H^{x}(x, y, 0)=0, H^{y}(x, y, 0)=$ $0, E^{z}(x, y, t)=J_{6}\left(\alpha_{6} r\right) \cos 6 \theta \cos \alpha_{6} t$, where $\alpha_{6}=13.589290170541217$ and $J_{6}(z)$ is the sixth Bessel function of the first kind. See [7].

We test three schemes in Section 2: DG on triangles, modified DG scheme and the curvilinear DG scheme for solving (1) at the final time $T=0.5$. See Table 1 for the errors in the electric filed on two meshes shown in Figure 4, where $L^{\infty}$ error is defined by the maximum of point-wise error over $\alpha$-optimized nodes [7] for all elements. The DG scheme on triangles is only second order accurate due to the homogeneous Dirichlet boundary condition imposed on the polygon boundary of the triangulation. Only the convex case as in shown in Figure 2 is 
involved thus we expect the modified DG scheme (7) and (8) to be high order accurate. We can see the modified DG scheme is indeed as accurate as the full curvilinear DG scheme.

Table 1: Example 1. $L^{\infty}$ error in DG schemes with polynomials of degree $N$ solving the TM-form Maxwell's equations on a unit disk.

\begin{tabular}{|c|ccc||ccc||ccc|}
\hline & \multicolumn{4}{|c||}{ DG on triangles } & \multicolumn{3}{|c||}{ modified DG } & \multicolumn{3}{|c|}{ curvilinear DG } & \\
\hline $\mathrm{N}$ & Mesh 1 & Mesh 2 & order & Mesh 1 & Mesh 2 & order & Mesh 1 & Mesh 2 & order \\
\hline 2 & $4.93 \mathrm{E}-2$ & $9.59 \mathrm{E}-3$ & 2.36 & $3.90 \mathrm{E}-2$ & $7.77 \mathrm{E}-3$ & 2.33 & $4.01 \mathrm{E}-2$ & $8.01 \mathrm{E}-3$ & 2.32 \\
\hline 3 & $1.85 \mathrm{E}-2$ & $3.03 \mathrm{E}-3$ & 2.61 & $1.20 \mathrm{E}-2$ & $8.12 \mathrm{E}-4$ & 3.90 & $1.31 \mathrm{E}-2$ & $8.04 \mathrm{E}-4$ & 4.03 \\
\hline 4 & $1.14 \mathrm{E}-2$ & $2.89 \mathrm{E}-3$ & 1.98 & $1.66 \mathrm{E}-3$ & $7.42 \mathrm{E}-5$ & 4.49 & $1.68 \mathrm{E}-3$ & $7.72 \mathrm{E}-5$ & 4.44 \\
\hline 5 & $1.11 \mathrm{E}-2$ & $2.66 \mathrm{E}-3$ & 2.06 & $3.06 \mathrm{E}-4$ & $4.68 \mathrm{E}-6$ & 6.03 & $3.20 \mathrm{E}-4$ & $4.70 \mathrm{E}-6$ & 6.09 \\
\hline
\end{tabular}

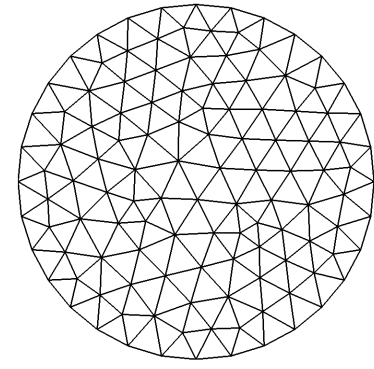

(a) Mesh 1

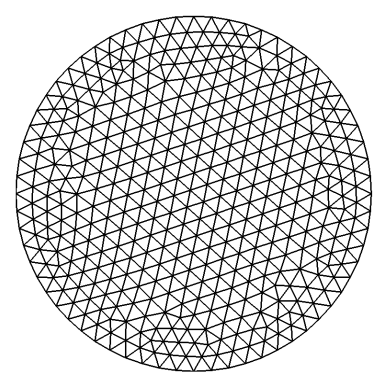

(b) Mesh 2

Figure 4: Two triangular meshes for a unit disk.

\section{Example 2.}

Consider two concentric PEC cylinders with an electromagnetic wave trapped between the walls in $[29,30]$. We solve $(22)$ in the domain between two concentric circles with radii $\frac{1}{6}$ and $\frac{1}{2}$. The boundary condition is $E^{z}=0$ along the boundary. The exact solution in polar coordinates $(r, \theta)$ is given by,

$$
\begin{aligned}
E^{z} & =\cos (\omega t+\theta)\left[J_{1}(\omega r)+a Y_{1}(\omega r)\right], \\
H^{x} & =-\frac{1}{2} \sin (\omega t+\theta) \sin \theta\left[J_{0}(\omega r)-J_{2}(\omega r)+a\left(Y_{0}(\omega r)-Y_{2}(\omega r)\right)\right]-\frac{\cos \theta}{\omega r} E^{z}, \\
H^{y} & =\frac{1}{2} \sin (\omega t+\theta) \cos \theta\left[J_{0}(\omega r)-J_{2}(\omega r)+a\left(Y_{0}(\omega r)-Y_{2}(\omega r)\right)\right]-\frac{\sin \theta}{\omega r} E^{z},
\end{aligned}
$$

where and $J_{n}$ and $Y_{n}$ denote the $n$-th order Bessel functions of the first and second kind, respectively. The constants are given by $\omega=9.813695999428405$ and $a=1.76368380110927$. 


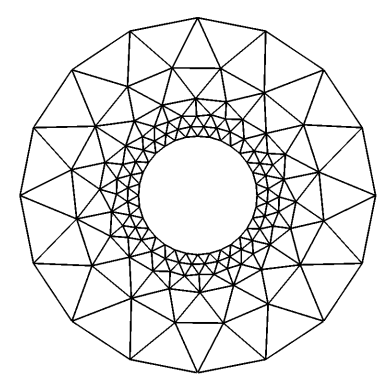

(a) Mesh 1

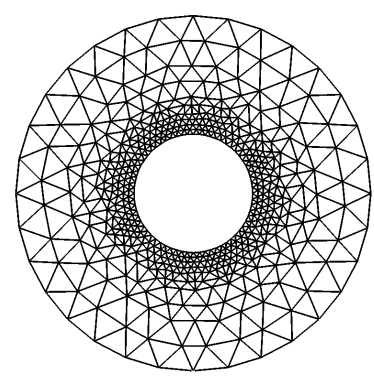

(b) Mesh 2

Figure 5: Two triangular meshes between two concentric circles with radii $\frac{1}{6}$ and $\frac{1}{2}$.

We test three DG schemes for solving (1) at the final time $T=0.5$. See Table 2 for the errors in the electric filed on two meshes shown in Figure 5. Notice that the concave case is present in the modified DG scheme and the exact solution can be smoothly extended to exterior of the domain. We can see the modified DG scheme is still as accurate as the full curvilinear DG scheme.

Table 2: Example 2. $L^{\infty}$ error in DG schemes with polynomials of degree $N$ solving the TM-form Maxwell's equations between two concentric circles.

\begin{tabular}{|c|ccc||ccc||ccc|}
\hline & \multicolumn{4}{|c||}{ DG on triangles } & \multicolumn{3}{|c||}{ modified DG } & \multicolumn{3}{c|}{ curvilinear DG } \\
\hline $\mathrm{N}$ & Mesh 1 & Mesh 2 & order & Mesh 1 & Mesh 2 & order & Mesh 1 & Mesh 2 & order \\
\hline 2 & $4.58 \mathrm{E}-2$ & $1.00 \mathrm{E}-2$ & 2.19 & $2.53 \mathrm{E}-2$ & $4.01 \mathrm{E}-3$ & 2.65 & $3.09 \mathrm{E}-2$ & $4.47 \mathrm{E}-3$ & 2.79 \\
\hline 3 & $3.73 \mathrm{E}-2$ & $8.87 \mathrm{E}-3$ & 2.07 & $3.37 \mathrm{E}-3$ & $3.20 \mathrm{E}-4$ & 3.39 & $3.41 \mathrm{E}-3$ & $2.77 \mathrm{E}-4$ & 3.62 \\
\hline 4 & $3.78 \mathrm{E}-2$ & $8.89 \mathrm{E}-3$ & 2.09 & $3.53 \mathrm{E}-4$ & $1.20 \mathrm{E}-5$ & 4.88 & $4.05 \mathrm{E}-4$ & $1.35 \mathrm{E}-5$ & 4.90 \\
\hline 5 & $3.79 \mathrm{E}-2$ & $8.92 \mathrm{E}-3$ & 2.09 & $2.45 \mathrm{E}-5$ & $6.92 \mathrm{E}-7$ & 5.15 & $3.15 \mathrm{E}-5$ & $6.74 \mathrm{E}-7$ & 5.54 \\
\hline
\end{tabular}

\section{2. $L D G$ for the Second Order Wave Equation}

\section{Example 3.}

By setting $u=E^{z}$ and $\nabla u=\left(H^{y},-H^{x}\right)$, the wave equation (14) with $c \equiv 1$ is equivalent to the system (22). Consider solving the wave equation (14) with $c \equiv 1$ on the unit disk with homogeneous Dirichlet boundary condition $u=0$ using the same initial condition as in Example 1. Then the exact solution is the same as in Example 1.

We test the modified LDG scheme and the curvilinear LDG scheme in Section 3 for solving (14) at the final time $T=0.5$. See Table 3 for the errors, where Mesh 1 and Mesh 2 are the same as in Figure 4, and Mesh 3 is a natural refinement of Mesh 2. We can see the modified LDG scheme is indeed as accurate as the full curvilinear LDG scheme. 


\section{Example 4.}

Consider solving the wave equation (14) with $c \equiv 1$ on the same domain as in Example 2 with homogeneous Dirichlet boundary condition $u=0$ using the same initial condition as in Example 2. Then the exact solution is the same as in Example 2. We test the modified LDG scheme and the curvilinear LDG scheme in Section 3 for solving (14) at the final time $T=0.5$. See Table 3 for the errors, where Mesh 1 and Mesh 2 are the same as in Figure 5, and Mesh 3 is a natural refinement of Mesh 2. We can see the modified LDG scheme is indeed as accurate as the full curvilinear LDG scheme.

Table 3: Example 3. $L^{\infty}$ error in LDG schemes with polynomials of degree $N$ solving the scalar wave equation on a unit disk.

\begin{tabular}{|c||ccccc||ccccc|}
\hline & \multicolumn{5}{|c||}{ modified LDG } & \multicolumn{5}{c|}{ curvilinear LDG } \\
\hline $\mathrm{N}$ & Mesh 1 & Mesh 2 & order & Mesh 3 & order & Mesh 1 & Mesh 2 & order & Mesh 3 & order \\
\hline 2 & $3.12 \mathrm{E}-2$ & $3.61 \mathrm{E}-3$ & 3.11 & $5.15 \mathrm{E}-4$ & 2.81 & $3.12 \mathrm{E}-2$ & $3.61 \mathrm{E}-3$ & 3.11 & $5.15 \mathrm{E}-4$ & 2.81 \\
\hline 3 & $9.53 \mathrm{E}-3$ & $8.58 \mathrm{E}-4$ & 3.47 & $5.77 \mathrm{E}-5$ & 3.90 & $8.40 \mathrm{E}-3$ & $8.60 \mathrm{E}-4$ & 3.29 & $5.77 \mathrm{E}-5$ & 3.90 \\
\hline 4 & $2.12 \mathrm{E}-3$ & $8.40 \mathrm{E}-5$ & 4.68 & $2.71 \mathrm{E}-6$ & 4.95 & $2.15 \mathrm{E}-3$ & $8.40 \mathrm{E}-5$ & 4.68 & $2.71 \mathrm{E}-6$ & 4.95 \\
\hline 5 & $3.58 \mathrm{E}-4$ & $7.93 \mathrm{E}-6$ & 5.50 & $1.22 \mathrm{E}-7$ & 6.03 & $3.62 \mathrm{E}-4$ & $7.97 \mathrm{E}-6$ & 5.50 & $1.22 \mathrm{E}-7$ & 6.03 \\
\hline
\end{tabular}

Table 4: Example 4. $L^{\infty}$ error in LDG schemes with polynomials of degree $N$ solving the scalar wave equation between two concentric circles.

\begin{tabular}{|c||ccccc||ccccc|}
\hline & \multicolumn{5}{|c||}{ modified LDG } & \multicolumn{5}{c|}{ curvilinear LDG } \\
\hline $\mathrm{N}$ & Mesh 1 & Mesh 2 & order & Mesh 3 & order & Mesh 1 & Mesh 2 & order & Mesh 3 & order \\
\hline 2 & $2.30 \mathrm{E}-3$ & $2.90 \mathrm{E}-3$ & 2.99 & $3.96 \mathrm{E}-5$ & 2.87 & $2.31 \mathrm{E}-3$ & $2.90 \mathrm{E}-4$ & 2.99 & $3.96 \mathrm{E}-5$ & 2.87 \\
\hline 3 & $6.27 \mathrm{E}-4$ & $4.35 \mathrm{E}-5$ & 3.85 & $3.14 \mathrm{E}-6$ & 3.79 & $5.62 \mathrm{E}-4$ & $4.36 \mathrm{E}-5$ & 3.69 & $3.14 \mathrm{E}-6$ & 3.79 \\
\hline 4 & $1.10 \mathrm{E}-4$ & $2.94 \mathrm{E}-6$ & 5.22 & $9.18 \mathrm{E}-8$ & 5.00 & $9.19 \mathrm{E}-5$ & $2.94 \mathrm{E}-6$ & 4.97 & $9.31 \mathrm{E}-8$ & 4.98 \\
\hline 5 & $1.68 \mathrm{E}-5$ & $2.16 \mathrm{E}-7$ & 6.28 & $4.22 \mathrm{E}-9$ & 5.68 & $1.07 \mathrm{E}-5$ & $2.15 \mathrm{E}-7$ & 5.64 & $4.22 \mathrm{E}-9$ & 5.67 \\
\hline
\end{tabular}

\subsection{Scalar Conservation Laws}

\section{Example 5.}

Consider an exact solution $u(x, y, t)=\sin (\pi(x+y-2 t))$ for the linear equation $u_{t}+u_{x}+u_{y}=0$. We construct an initial-boundary value problem on a unit disk by first setting the initial condition as $u_{0}(x, y)=\sin (\pi(x+y))$. Since the flow direction is $(1,1)$, the inflow boundary is $\Gamma=\left\{(x, y): x^{2}+y^{2}=1, x+y \leq 0\right\}$. The exact solution $u(x, y, t)=\sin (\pi(x+y-2 t))$ on $\Gamma$ is given as the boundary condition.

We test the modified DG, modified DG with the conservation correction and curvilinear DG schemes till $T=1$ on the two meshes in Figure 4. Since a fourth order Runge-Kutta is used for time discretization, the time step is taken as $\Delta t=\mathcal{O}\left(h^{\frac{N+1}{4}}\right)$ if polynomial degree $N$ is larger than 3 , where $h$ is the mesh 
size. See Table 5 for the errors. The simple conservation correction step is used for each time stage in the low-storage fourth order Runge-Kutta. The error of modified DG with this conservation correction is a bit larger than modified DG without the correction.

Table 5: Example 5. $L^{\infty}$ error in DG schemes with polynomials of degree $N$ solving $2 \mathrm{D}$ linear equation on a unit disk.

\begin{tabular}{|c||ccc||ccc||ccc|}
\hline \multicolumn{1}{|||}{} & \multicolumn{3}{|c||}{$\begin{array}{l}\text { modified DG with } \\
\text { conservation correction }\end{array}$} & \multicolumn{2}{|c||}{ modified DG } & & \multicolumn{3}{c|}{ curvilinear DG } \\
\hline $\mathrm{N}$ & Mesh 1 & Mesh 2 & order & Mesh 1 & Mesh 2 & order & Mesh 1 & Mesh 2 & order \\
\hline 2 & $2.15 \mathrm{E}-2$ & $3.19 \mathrm{E}-3$ & 2.75 & $1.95 \mathrm{E}-2$ & $2.75 \mathrm{E}-3$ & 2.83 & $2.11 \mathrm{E}-2$ & $2.96 \mathrm{E}-3$ & 2.83 \\
\hline 3 & $1.90 \mathrm{E}-3$ & $1.04 \mathrm{E}-4$ & 4.19 & $1.90 \mathrm{E}-3$ & $9.97 \mathrm{E}-5$ & 4.25 & $1.47 \mathrm{E}-3$ & $1.03 \mathrm{E}-4$ & 3.83 \\
\hline 4 & $1.03 \mathrm{E}-4$ & $3.52 \mathrm{E}-6$ & 4.88 & $1.03 \mathrm{E}-4$ & $3.27 \mathrm{E}-6$ & 4.98 & $9.67 \mathrm{E}-5$ & $3.54 \mathrm{E}-6$ & 4.77 \\
\hline 5 & $5.96 \mathrm{E}-6$ & $7.10 \mathrm{E}-8$ & 6.39 & $5.94 \mathrm{E}-6$ & $7.00 \mathrm{E}-8$ & 6.41 & $3.86 \mathrm{E}-6$ & $7.03 \mathrm{E}-8$ & 5.78 \\
\hline
\end{tabular}

\section{Example 6.}

Let $u(x, y, t)$ be the exact solution to $2 \mathrm{D}$ Burgers' equation $u_{t}+u u_{x}+u u_{y}=0$ with the initial condition $u_{0}(x, y)=1+\frac{1}{2} \sin (\pi(x+y-2 t))$. Then the flow direction of this solution is $(1,1)$ before shock waves emerge.

We construct an initial-boundary value problem on a unit disk by using $u(x, y, 0)$ as the initial condition and using $u(x, y, t)$ as boundary condition on the inflow boundary $\Gamma=\left\{(x, y): x^{2}+y^{2}=1, x+y \leq 0\right\}$.

We test the modified DG, modified DG with the conservation correction and curvilinear DG schemes till $T=0.05$ on the two meshes in Figure 4. The time step is taken as $\Delta t=\mathcal{O}\left(h^{\frac{N+1}{4}}\right)$ if polynomial degree $N$ is larger than 3, where $h$ is the mesh size. See Table 6 for the errors.

Table 6: Example 6. $L^{\infty}$ error in DG schemes with polynomials of degree $N$ solving 2D Burgers' equation on a unit disk.

\begin{tabular}{|c||ccc||ccc||ccc|}
\hline \multicolumn{1}{||}{} & \multicolumn{3}{|c||}{ modified DG with } & \multicolumn{3}{c||}{ modified DG } & \multicolumn{3}{c|}{ curvilinear DG } \\
\hline $\mathrm{N}$ & conservation correction & & & & & & & & \\
\hline 2 & $1.38 \mathrm{E}-2$ & $2.48 \mathrm{E}-3$ & 2.48 & $1.38 \mathrm{E}-2$ & $2.49 \mathrm{E}-3$ & 2.47 & $1.38 \mathrm{E}-2$ & $2.53 \mathrm{E}-3$ & 2.46 \\
\hline 3 & $2.77 \mathrm{E}-3$ & $1.80 \mathrm{E}-4$ & 3.94 & $2.78 \mathrm{E}-3$ & $1.79 \mathrm{E}-4$ & 3.95 & $2.84 \mathrm{E}-3$ & $1.79 \mathrm{E}-4$ & 3.98 \\
\hline 4 & $2.83 \mathrm{E}-4$ & $1.53 \mathrm{E}-5$ & 4.21 & $2.83 \mathrm{E}-4$ & $1.53 \mathrm{E}-5$ & 4.21 & $3.87 \mathrm{E}-4$ & $1.53 \mathrm{E}-5$ & 4.66 \\
\hline 5 & $6.23 \mathrm{E}-5$ & $1.01 \mathrm{E}-6$ & 5.95 & $6.27 \mathrm{E}-5$ & $1.00 \mathrm{E}-6$ & 5.96 & $6.13 \mathrm{E}-5$ & $1.00 \mathrm{E}-6$ & 5.93 \\
\hline
\end{tabular}

\subsection{Compressible Euler Equations}

Consider the two-dimensional Euler equations for the ideal gas

$$
\frac{\partial}{\partial t}\left(\begin{array}{c}
\rho \\
\rho u \\
\rho v \\
E
\end{array}\right)+\frac{\partial}{\partial x}\left(\begin{array}{c}
\rho u \\
\rho u^{2}+p \\
\rho u v \\
(E+p) u
\end{array}\right)+\frac{\partial}{\partial y}\left(\begin{array}{c}
\rho v \\
\rho u v \\
\rho v^{2}+p \\
(E+p) v
\end{array}\right)=0
$$




$$
p=(\gamma-1)\left(E-\frac{1}{2} \rho u^{2}-\frac{1}{2} \rho v^{2}\right),
$$

where $\gamma=1.4$.

\section{Example 7.}

An exact solution with constant entropy is given by $\rho=1+\frac{1}{2} \sin (2 \pi(x+y-2 t))$ and $u=v=p \equiv 1$. Let $\mathbf{w}(x, y, t)$ denote this exact solution. We construct an initial-boundary value problem on a unit disk by using $\mathbf{w}(x, y, 0)$ as the initial condition and using $\mathbf{w}(x, y, t)$ as boundary condition on the inflow boundary $\Gamma=\left\{(x, y): x^{2}+y^{2}=1, x+y \leq 0\right\}$.

We test the modified DG, modified DG with the conservation correction and curvilinear DG schemes till $T=0.2$ on the two meshes in Figure 4. The time step is taken as $\Delta t=\mathcal{O}\left(h^{\frac{N+1}{4}}\right)$ if polynomial degree $N$ is larger than 3, where $h$ is the mesh size. See Table 7 for the errors.

Table 7: Example 7. $L^{\infty}$ error for density in DG schemes with polynomials of degree $N$ solving 2D Euler's equations on a unit disk.

\begin{tabular}{|c||ccc||ccc||ccc|}
\hline \multicolumn{1}{|l||}{} & \multicolumn{3}{|c||}{$\begin{array}{c}\text { modified DG with } \\
\text { conservation fix }\end{array}$} & \multicolumn{3}{c||}{ modified DG } & & \multicolumn{3}{c|}{ curvilinear DG } \\
\hline $\mathrm{N}$ & Mesh 1 & Mesh 2 & order & Mesh 1 & Mesh 2 & order & Mesh 1 & Mesh 2 & order \\
\hline 2 & $6.20 \mathrm{E}-2$ & $8.74 \mathrm{E}-3$ & 2.83 & $6.20 \mathrm{E}-2$ & $9.76 \mathrm{E}-3$ & 2.67 & $6.21 \mathrm{E}-2$ & $8.96 \mathrm{E}-3$ & 2.84 \\
\hline 3 & $1.13 \mathrm{E}-2$ & $5.83 \mathrm{E}-4$ & 4.28 & $1.13 \mathrm{E}-2$ & $5.82 \mathrm{E}-4$ & 4.28 & $1.13 \mathrm{E}-2$ & $5.95 \mathrm{E}-4$ & 4.25 \\
\hline 4 & $1.24 \mathrm{E}-3$ & $4.22 \mathrm{E}-5$ & 4.88 & $1.24 \mathrm{E}-3$ & $4.22 \mathrm{E}-5$ & 4.88 & $1.15 \mathrm{E}-3$ & $4.19 \mathrm{E}-5$ & 4.77 \\
\hline 5 & $1.32 \mathrm{E}-4$ & $1.51 \mathrm{E}-6$ & 6.45 & $1.31 \mathrm{E}-4$ & $1.51 \mathrm{E}-6$ & 6.45 & $1.65 \mathrm{E}-4$ & $1.86 \mathrm{E}-6$ & 6.47 \\
\hline
\end{tabular}

\section{Example 8.}

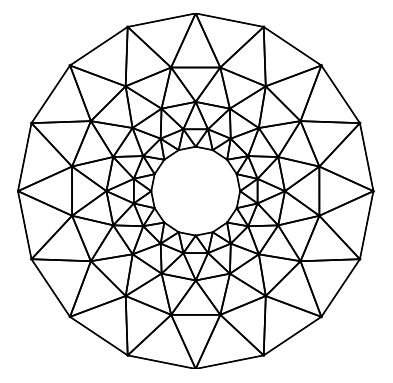

(a) Mesh 1

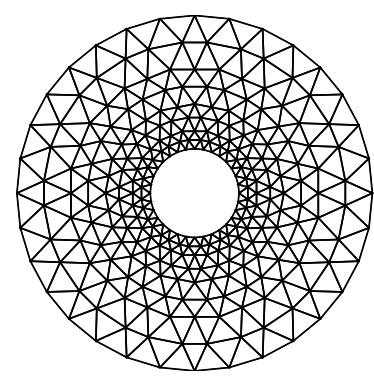

(b) Mesh 2

Figure 6: Two triangular meshes for the Couette Flow problem. 
Consider the Couette Flow test problem in [7] for the flow between two concentric cylinders spinning at different velocities. The exact solution in polar coordinates $(r, \theta)$ is given by

$$
\begin{aligned}
\rho & =1, \\
\rho u & =-\frac{\sin \theta}{75}\left(-r+\frac{16}{r}\right), \\
\rho v & =\frac{\cos \theta}{75}\left(-r+\frac{16}{r}\right), \\
p & =1+\frac{1}{75^{2}}\left(\frac{r^{2}}{2}-32 \ln r-\frac{128}{r^{2}}\right) .
\end{aligned}
$$

The computational domain is the area between two concentric circles with radii $r=1$ and $r=4$. The exact solution is used as the initial condition and boundary conditions on two circles. We test the modified DG and curvilinear DG schemes till $T=0.2$ on the two meshes in Figure 6. Since the exact solution can be smoothly extended, we expect the modified DG to be high order accurate. It is indeed high order accurate as shown in Table 8 , where $L^{1}$ error is defined as the arithmetic average of point-wise error over $\alpha$-optimized nodes for all elements.

Table 8: Example 8. $L^{1}$ error for density in DG schemes with polynomials of degree $N$ for Couette Flow.

\begin{tabular}{|c||ccc||ccc||ccc|}
\hline \multicolumn{1}{||||}{} & \multicolumn{3}{|c||}{$\begin{array}{l}\text { modified DG with } \\
\text { conservation fix }\end{array}$} & \multicolumn{3}{|c||}{ modified DG } & & \multicolumn{3}{c|}{ curvilinear DG } \\
\hline $\mathrm{N}$ & Mesh 1 & Mesh 2 & order & Mesh 1 & Mesh 2 & order & Mesh 1 & Mesh 2 & order \\
\hline 2 & $1.73 \mathrm{E}-4$ & $1.89 \mathrm{E}-5$ & 3.20 & $1.74 \mathrm{E}-4$ & $1.89 \mathrm{E}-5$ & 3.20 & $1.53 \mathrm{E}-4$ & $1.71 \mathrm{E}-5$ & 3.16 \\
\hline 3 & $2.01 \mathrm{E}-5$ & $1.11 \mathrm{E}-6$ & 4.23 & $2.08 \mathrm{E}-5$ & $1.11 \mathrm{E}-6$ & 4.23 & $1.76 \mathrm{E}-5$ & $1.04 \mathrm{E}-6$ & 4.08 \\
\hline 4 & $1.38 \mathrm{E}-6$ & $5.89 \mathrm{E}-8$ & 4.55 & $1.38 \mathrm{E}-6$ & $5.90 \mathrm{E}-8$ & 4.55 & $1.36 \mathrm{E}-6$ & $5.95 \mathrm{E}-8$ & 4.52 \\
\hline 5 & $2.82 \mathrm{E}-7$ & $5.93 \mathrm{E}-9$ & 5.57 & $2.82 \mathrm{E}-7$ & $5.94 \mathrm{E}-9$ & 5.57 & $2.11 \mathrm{E}-7$ & $5.66 \mathrm{E}-9$ & 5.22 \\
\hline
\end{tabular}

\section{Example 9.}

One important desired property for numerical methods on curved grids is free-stream preservation, i.e., the ability to exactly preserve a constant-state solution. Assume $u_{h} \equiv c$ is a constant, then the consistency of the numerical flux implies $\widehat{\mathbf{F} \cdot \mathbf{n}} \equiv \mathbf{F}(c) \cdot \mathbf{n}$. Thus after plugging $u_{h} \equiv c$, we obtain $\iint_{\widetilde{K}} \frac{\partial}{\partial t} u_{h} v_{h} d \mathbf{x}=$ 0 in (3) and $\iint_{K} \frac{\partial}{\partial t} u_{h} v_{h} d \mathbf{x}=0$ (8). Therefore free-stream preservation is a trivial property satisfied by both curvilinear DG scheme (3) and the modified scheme (8). Moreover, the conservation correction step discussed in Section 4 does not destroy the free-stream preservation property because such a correction will be zero when $u_{h} \equiv c$.

We test the free stream preservation property of the modified DG scheme solving a constant solution of compressible Euler equations on the two meshes 
in Figure 6. The exact solution has constant unit density and pressure. A freestream of Mach number 0.5 in $x$-direction is imposed. The $y$-direction velocity $v$ is zero. The error for the $y$-direction velocity $v$ at terminal time $T=10$ is listed in Table 9. In all three schemes, the numerical solution for $v$ is close to machine zero.

Table 9: Example 9. Free-stream preservation test. $L^{\infty}$ error for $v$ in DG schemes with polynomials of degree $N$.

\begin{tabular}{|c||cc||cc||cc|}
\hline \multicolumn{1}{||||}{} & \multicolumn{2}{c||}{$\begin{array}{c}\text { modified DG with } \\
\text { conservation fix }\end{array}$} & \multicolumn{2}{c||}{ modified DG } & \multicolumn{2}{c|}{ curvilinear DG } \\
\hline $\mathrm{N}$ & Mesh 1 & Mesh 2 & Mesh 1 & Mesh 2 & Mesh 1 & Mesh 2 \\
\hline 2 & $2.39 \mathrm{E}-14$ & $3.08 \mathrm{E}-14$ & $2.02 \mathrm{E}-15$ & $1.84 \mathrm{E}-15$ & $2.25 \mathrm{E}-15$ & $3.09 \mathrm{E}-15$ \\
\hline 3 & $2.29 \mathrm{E}-14$ & $3.33 \mathrm{E}-14$ & $2.98 \mathrm{E}-15$ & $3.80 \mathrm{E}-15$ & $3.87 \mathrm{E}-15$ & $4.53 \mathrm{E}-15$ \\
\hline 4 & $1.82 \mathrm{E}-14$ & $4.20 \mathrm{E}-14$ & $7.26 \mathrm{E}-15$ & $1.05 \mathrm{E}-14$ & $8.08 \mathrm{E}-15$ & $8.66 \mathrm{E}-15$ \\
\hline 5 & $2.06 \mathrm{E}-14$ & $5.10 \mathrm{E}-14$ & $7.06 \mathrm{E}-15$ & $2.62 \mathrm{E}-14$ & $1.01 \mathrm{E}-14$ & $2.70 \mathrm{E}-14$ \\
\hline
\end{tabular}

\subsection{Reflective Boundary Conditions}

\section{Example 10.}

Consider the following acoustic wave equations:

$$
\begin{array}{r}
\rho(\mathbf{x}) \frac{\partial \mathbf{v}}{\partial t}+\nabla p=\mathbf{0}, \\
\frac{1}{\kappa(\mathbf{x})} \frac{\partial p}{\partial t}+\nabla \cdot \mathbf{v}=0,
\end{array}
$$

where $\rho$ is density and $\kappa$ is bulk modulus. To test three DG schemes in Section 2 on wall boundary conditions, the Problem 2 in Category 1 of the Second CAA Workshop on Benchmark Problems [31] is studied, see also [8, 32]. The domain is a square $[-10,10] \times[-10,10]$ minus a disk of raidus 0.5 centered at the origin. The initial condition is

$$
\mathbf{v}=\mathbf{0}, \quad p=\exp \left[-\ln 2 \frac{(x-4)^{2}+y^{2}}{0.2^{2}}\right] .
$$

The linear system (23) with $\rho=\kappa \equiv 1$ is solved with reflective boundary conditions enforced on the circle, modeling the scattering of a Gaussian pressure pulse by a cylinder. Here reflective boundary conditions mean that the boundary condition of the normal velocity should be given as the opposite one in numerical solution at the boundary while the tangential velocity and pressure should be given as the same of those in numerical solution at the boundary.

See Figure 7 (a) and (b) for the time history of pressure monitored at the point $(0,5)$ for three DG schemes with cubic polynomials on the mesh shown in Figure 7 (c). The maximum of the reference solution in Figure 7 at $T=8.2$ corresponds to the scattered wave field. In the zoomed Figure 7 (b), we can 
observe that the polygonal approximation to the circle in the DG scheme on triangles results in an obvious phase error. The concave case in Figure 2 is present in the modified DG scheme but the exact solution cannot be smoothly extended due to the reflective boundary condition. Thus the modified DG scheme is not high order accurate for this problem. We can observe that the modified DG scheme is less accurate than the curvilinear DG scheme, however, the modified DG scheme does produce a smaller phase error than the DG scheme on triangles.

\section{Concluding Remarks}

We have discussed a modified DG scheme defined on a geometry fitting triangulation for solving time dependent problems on a two-dimensional curved domain. It can be easily implemented as a DG scheme defined on triangles with a modification or correction, which is simple in the sense that integrals over curved elements are avoided. The modified scheme is high order accurate on a triangle of convex case, and on a triangle of concave case if the equations and the exact smooth solution can be smoothly extended to the whole triangle. When such a smooth extension does not exist in a concave case, e.g., imposing reflective boundary conditions for flow passing a cylinder, the modified DG scheme produces smaller error than the DG scheme on triangles, even though it does not have a better convergence rate. However, such a modified scheme is not stable on a very coarse mesh. Rigorous stability result is difficult to establish on a fine enough mesh. Nonetheless, numerical tests suggest that the modified DG scheme is stable on a reasonably coarse mesh and finer ones.

\section{Appendix A.}

We discuss a one-dimensional analog of the modified DG scheme for the linear problem

$$
\left\{\begin{aligned}
u_{t}+u_{x} & =0, & & x \in[0,1], \\
u(x, 0) & =u_{0}(x), & & x \in[0,1], \\
u(x, t) & =0, & & x=0 .
\end{aligned}\right.
$$

Consider a mesh which may not fit the domain $[0,1]$ exactly, as illustrated in Figure A.8. Let $a=x_{\frac{1}{2}}<x_{\frac{3}{2}}<\cdots<x_{j-\frac{1}{2}}<x_{j+\frac{1}{2}}<\cdots<x_{\frac{N+1}{2}}=1$ for the interval $[a, 1]$ where $a \geq 0$.

Let $u_{j}(x, t)$ and $v_{j}(x)$ denote the solution polynomial and test function polynomial on the interval $I_{j}=\left[x_{j-\frac{1}{2}}, x_{j+\frac{1}{2}}\right]$. Let $v_{j+\frac{1}{2}}^{-}$denote $v_{j}\left(x_{j+\frac{1}{2}}\right)$ and $v_{j-\frac{1}{2}}^{+}$ denote $v_{j}\left(x_{j-\frac{1}{2}}\right)$. Then the strong formulation of DG scheme on $I_{j}$ is given by

$$
\int_{I_{j}}\left(\frac{\partial}{\partial t} u_{j}+\frac{\partial}{\partial x} u_{j}\right) v_{j} d x+\left(\widehat{u}_{j+\frac{1}{2}}-u_{j+\frac{1}{2}}^{-}\right) v_{j+\frac{1}{2}}^{-}-\left(\widehat{u}_{j-\frac{1}{2}}-u_{j-\frac{1}{2}}^{+}\right) v_{j-\frac{1}{2}}^{+}=0,
$$

where $\widehat{u}_{j+\frac{1}{2}}$ is the numerical flux approximating $u$ at $x_{j+\frac{1}{2}}$. For instance, we can use the upwind flux $\widehat{u}_{j+\frac{1}{2}}=u_{j+\frac{1}{2}}^{-}$for $j \geq 1$. The boundary condition should be 


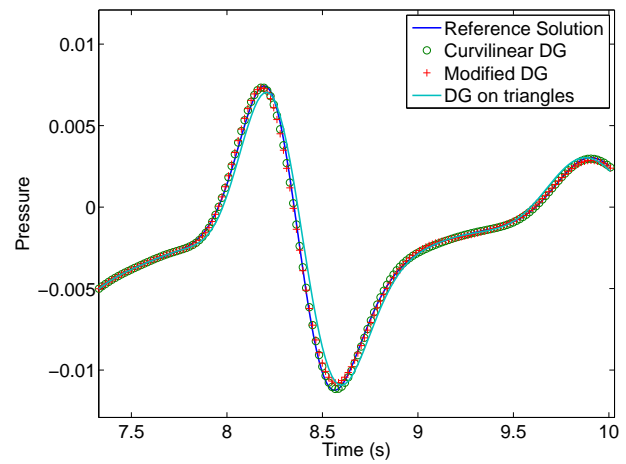

(a) Pressure at the point $(0,5)$.

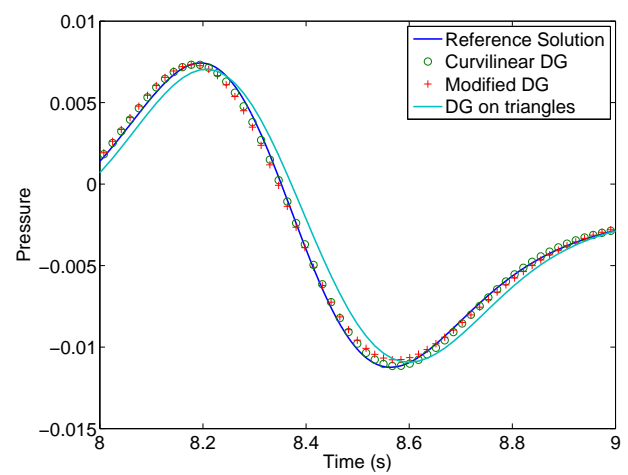

(b) Pressure at the point $(0,5)$.

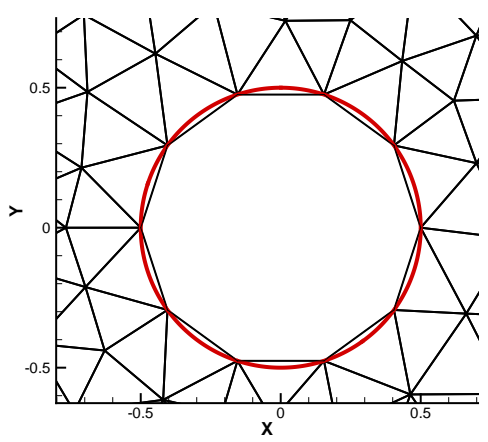

(c) Mesh

Figure 7: Acoustic Scattering by a Cylinder. DG with $P^{3}$ basis. 


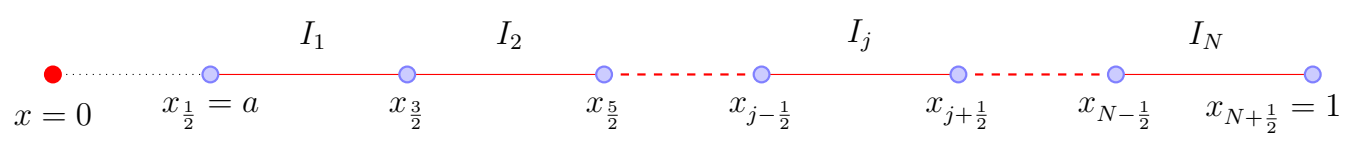

Figure A.8: An illustration of the mesh in one dimension.

also included in the scheme. If the mesh boundary exactly fits the true boundary, i.e., $a=0$ in Figure A.8, then one can take $\widehat{u}_{\frac{1}{2}}=u(0, t)=0$ in the classical DG scheme. Plugging in the numerical flux and setting $v_{j}=u_{j}$ in the DG scheme (A.2) for $j>1$, with the triangle inequality $u_{j-\frac{1}{2}}^{-} u_{j-\frac{1}{2}}^{+} \leq \frac{1}{2}\left(u_{j-\frac{1}{2}}^{-}\right)^{2}+\frac{1}{2}\left(u_{j-\frac{1}{2}}^{+}\right)^{2}$, we obtain

$$
\begin{aligned}
\int_{I_{j}} \frac{1}{2} \frac{\partial}{\partial t} u_{j}^{2} d x & =-\int_{I_{j}} \frac{1}{2} \frac{\partial}{\partial x} u_{j}^{2} d x+\left(u_{j-\frac{1}{2}}^{-}-u_{j-\frac{1}{2}}^{+}\right) u_{j-\frac{1}{2}}^{+} \\
& =\frac{1}{2}\left(u_{j-\frac{1}{2}}^{+}\right)^{2}-\frac{1}{2}\left(u_{j+\frac{1}{2}}^{-}\right)^{2}+\left(u_{j-\frac{1}{2}}^{-}-u_{j-\frac{1}{2}}^{+}\right) u_{j-\frac{1}{2}}^{+} \\
& =-\frac{1}{2}\left(u_{j-\frac{1}{2}}^{+}\right)^{2}-\frac{1}{2}\left(u_{j+\frac{1}{2}}^{-}\right)^{2}+u_{j-\frac{1}{2}}^{-} u_{j-\frac{1}{2}}^{+} \\
& \leq \frac{1}{2}\left(u_{j-\frac{1}{2}}^{-}\right)^{2}-\frac{1}{2}\left(u_{j+\frac{1}{2}}^{-}\right)^{2} .
\end{aligned}
$$

At the boundary cell $I_{1}$, plugging in the numerical flux $\widehat{u}_{\frac{1}{2}}=0$ and setting $v_{1}=u_{1}$, we get

$$
\int_{I_{1}} \frac{1}{2} \frac{\partial}{\partial t} u_{1}^{2} d x=-\int_{I_{1}} \frac{1}{2} \frac{\partial}{\partial x} u_{1}^{2} d x+\left(-u_{\frac{1}{2}}^{+}\right) u_{\frac{1}{2}}^{+}=-\frac{1}{2}\left(u_{\frac{1}{2}}^{+}\right)^{2}-\frac{1}{2}\left(u_{\frac{3}{2}}^{-}\right)^{2} .
$$

Summing (A.4) and (A.3) for $j=2, \cdots, N$, we obtain the $L^{2}$-stability of the classical DG scheme $\frac{\partial}{\partial t} \int_{0}^{1} u_{h}^{2}(x, t) d x \leq-\left(u_{\frac{1}{2}}^{+}\right)^{2}-\left(u_{N+\frac{1}{2}}^{-}\right)^{2} \leq 0$, where $u_{h}$ denotes the DG solution.

Now consider the case when the mesh boundary does not fit the true boundary, i.e., $a>0$. For cells $I_{j}$ with $j>1$ which are not adjacent to the boundary $x=0$, the DG scheme is still defined as in (A.2) and the inequality (A.3) still holds. For the boundary cell $I_{1}$, since the boundary condition is not given for the computational domain, a one-dimensional modified DG scheme can be defined as

$$
\int_{I_{1}}\left(\frac{\partial}{\partial t} u_{1}+\frac{\partial}{\partial x} u_{1}\right) v_{1} d x+\left(\widehat{u}_{\frac{3}{2}}-u_{\frac{3}{2}}^{-}\right) v_{\frac{3}{2}}^{-}-\left(\widehat{u}_{0}-u_{0}^{+}\right) v_{0}^{+}=0
$$

where $u_{0}^{+}$and $v_{0}^{+}$are the point values of $u_{1}(x, t)$ and $v_{1}(x)$ at $x=0$. For the numerical flux and boundary condition, we can take $\widehat{u}_{0}=u(0, t)=0$ and $\widehat{u}_{\frac{3}{2}}=u_{\frac{3}{2}}^{-}$. Setting $v_{1}=u_{1}$ in (A.5), we get

$$
\int_{I_{1}} \frac{1}{2} \frac{\partial}{\partial t} u_{1}^{2} d x=-\int_{I_{1}} \frac{1}{2} \frac{\partial}{\partial x} u_{1}^{2} d x-\left(u_{0}^{+}\right)^{2}=\frac{1}{2}\left(u_{\frac{1}{2}}^{+}\right)^{2}-\frac{1}{2}\left(u_{\frac{3}{2}}^{-}\right)^{2}-\left(u_{0}^{+}\right)^{2} .
$$


Summing (A.6) and (A.3) for $j=2, \cdots, N$, the L2-norm of the solution in the modified DG scheme (A.5) satisfies

$$
\frac{\partial}{\partial t} \int_{0}^{1} u_{h}^{2}(x, t) d x \leq\left(u_{\frac{1}{2}}^{+}\right)^{2}-2\left(u_{0}^{+}\right)^{2}-\left(u_{N+\frac{1}{2}}^{-}\right)^{2} .
$$

We can see that the $L^{2}$ norm of the solution in the modified DG scheme will blow up if $x_{\frac{1}{2}}=a$ is far away from $x=0$. Since (A.6) is an equality, the $L^{2}$ estimate above is sharp. Even if assuming $a$ is very close to 0 , it is highly nontrivial to prove an energy stability due to the positive term in the right hand side of (A.7).

\section{Appendix B.}

For linear equations, the modified DG scheme is linear. Any linear semidiscrete scheme for first order time-dependent problems can be represented as $\frac{d}{d t} \mathbf{u}_{h}=\mathcal{L}_{h} \mathbf{u}_{h}$ where $\mathbf{u}_{h}$ denotes all degree freedoms in the numerical solution and $\mathcal{L}_{h}$ is a linear operator denoting spatial discretization. We adopt a standard spectrum analysis of $\mathcal{L}_{h}$ to provide necessary but not sufficient conditions on stability of the modified DG scheme. If any of the eigenvalues of $\mathcal{L}_{h}$ has positive real part, then the scheme is not stable in the sense that it admits a solution with exponential growth in time.

We first discuss a simplified one-dimensional case. We only consider the modified DG scheme defined on a mesh consisting of only one interval $I=[0,1]$ for solving the initial-boundary value problem

$$
\left\{\begin{aligned}
u_{t}+u_{x} & =0, & & x \in[-a, 1], \\
u(x, 0) & =u_{0}(x), & & x \in[-a, 1], \\
u(x, t) & =0, & & x=-a,
\end{aligned}\right.
$$

where $0 \leq a \leq 1$. We are interested in what values of $a$ may result in positive real parts in the eigenvalues of spatial discretization operator $\mathcal{L}_{h}$ in the modified scheme. Let $\max \operatorname{Re}\left(\operatorname{eig}\left(\mathcal{L}_{h}\right)\right)$ denote the largest real parts in the eigenvalues of $\mathcal{L}_{h}$. See Figure B.9 for $\max \operatorname{Re}\left(\operatorname{eig}\left(\mathcal{L}_{h}\right)\right)$ for the modified DG scheme with $P^{5}$ and $P^{8}$ polynomials, which suggests that the modified DG scheme is not stable for large misfit between mesh boundary and true boundary. We can see that the $\max \operatorname{Re}\left(\operatorname{eig}\left(\mathcal{L}_{h}\right)\right)$ is monotonically increasing with respect to $a$ when $\max \operatorname{Re}\left(\operatorname{eig}\left(\mathcal{L}_{h}\right)\right) \leq 0$. Figure B.10 is a graph of the largest $a \in[0,1]$ such that $\max \operatorname{Re}\left(\operatorname{eig}\left(\mathcal{L}_{h}\right)\right) \leq 0$ for the modified DG scheme using $P^{N}$ polynomial.

Next we discuss a simplified two-dimensional case. Consider a domain $\Omega$ as illustrated in Figure B.11, where the origin is denoted by $O$ and $\theta \in\left[0, \frac{\pi}{2}\right]$ is the angle between $A O$ and $B O$. Let $A C$ and $B C$ be two segments parallel to two coordinates axes respectively and $A B$ be the arc lying on the unit circle. Then the coordinates of $A, B$ and $C$ are $\left(-\cos \left(\frac{\pi}{4}-\frac{\theta}{2}\right),-\sin \left(\frac{\pi}{4}-\frac{\theta}{2}\right)\right),\left(-\sin \left(\frac{\pi}{4}-\right.\right.$ $\left.\left.\frac{\theta}{2}\right),-\cos \left(\frac{\pi}{4}-\frac{\theta}{2}\right)\right)$ and $\left(-\sin \left(\frac{\pi}{4}-\frac{\theta}{2}\right),-\sin \left(\frac{\pi}{4}-\frac{\theta}{2}\right)\right)$. Consider the modified DG 


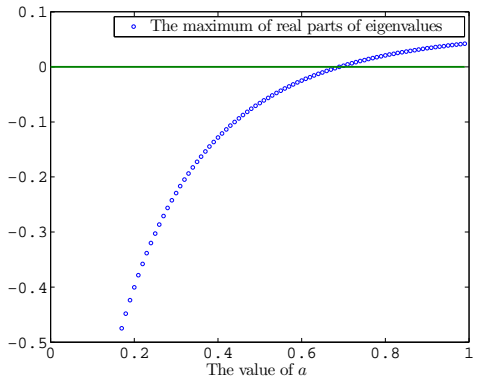

(a) polynomials of degree 5 basis.

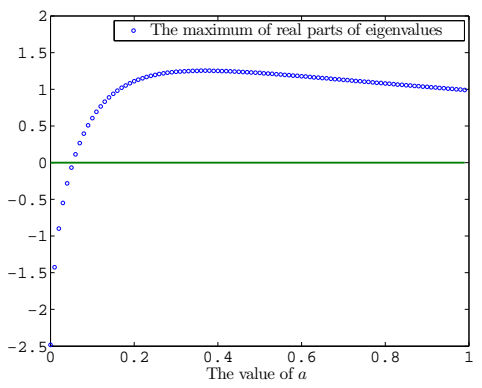

(b) polynomials of degree 8 basis.

Figure B.9: The maximum of real parts in the eigenvalues of spatial discretization operator $\mathcal{L}_{h}$ in the $1 \mathrm{D}$ modified DG scheme, defined on a mesh consisting of one interval $[0,1]$ for solving (B.1).

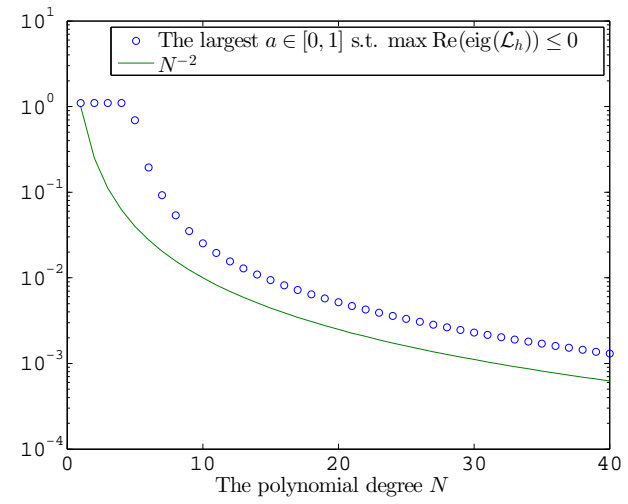

Figure B.10: The largest $a \in[0,1]$ such that $\max \operatorname{Re}\left(\operatorname{eig}\left(\mathcal{L}_{h}\right)\right) \leq 0$ in the $1 \mathrm{D}$ modified DG scheme with polynomials of degree $N$ basis, defined on a mesh consisting of one interval $[0,1]$ for solving (B.1). The decay rate fits $\mathcal{O}\left(\frac{1}{N^{2}}\right)$ quite well. 
scheme defined on the triangle $A B C$ solving the linear problem

$$
\left\{\begin{aligned}
u_{t}+u_{x}+u_{y} & =0, & & \mathbf{x} \in \Omega, \\
u(\mathbf{x}, 0) & =u_{0}(\mathbf{x}), & & \mathbf{x} \in \Omega, \\
u(\mathbf{x}, t) & =0, & & \mathbf{x} \in \Gamma,
\end{aligned}\right.
$$

where the inflow boundary $\Gamma$ is the arc $A B$ as illustrated in Figure B.11. The smaller $\theta$ is, the closer the segment $A B$ is to the unit circle. Thus the value of $\theta$ here is used as a quantification of misfit between the mesh boundary and the true boundary. See Figure B.12 for largest real parts of the eigenvalues for the modified DG scheme with polynomials of degree 5 . We observe that $\max \operatorname{Re}\left(\operatorname{eig}\left(\mathcal{L}_{h}\right)\right)$ is monotonically increasing with respect to $\theta$ when $\max \operatorname{Re}\left(\operatorname{eig}\left(\mathcal{L}_{h}\right)\right) \leq 0$. The largest angles $\theta$ such that $\max \operatorname{Re}\left(\operatorname{eig}\left(\mathcal{L}_{h}\right)\right) \leq 0$ are listed in Table B.10.

Table B.10: The largest angles $\theta$ such that $\max \operatorname{Re}\left(\operatorname{eig}\left(\mathcal{L}_{h}\right)\right) \leq 0$ in $2 \mathrm{D}$ modified DG scheme with polynomials of degree $N$ defined on the triangle $A B C$ in Figure B.11.

\begin{tabular}{|c||c|c|c|c|c|c|c|c|}
\hline$N$ & 2 & 3 & 4 & 5 & 6 & 7 & 8 & 9 \\
\hline$\theta$ & $90^{\circ}$ & $90^{\circ}$ & $90^{\circ}$ & $81.97^{\circ}$ & $25.39^{\circ}$ & $12.05^{\circ}$ & $6.86^{\circ}$ & $4.38^{\circ}$ \\
\hline
\end{tabular}

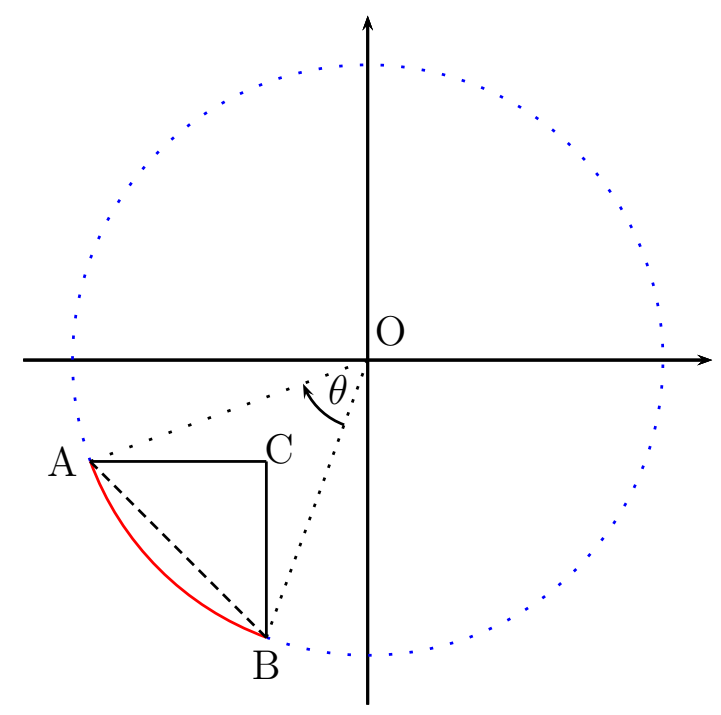

Figure B.11: The domain $\Omega$ is bounded by the arc $A B$ and two segments $A C$ and $B C$. The inflow boundary $\Gamma$ is the arc $A B$.

\section{Acknowledgments}

The author is grateful to Krzysztof Fidkowski, David Kopriva, Chi-Wang $\mathrm{Shu}$, Sirui Tan and Zhengfu Xu for numerous insightful discussions. The research was supported in part by the NSF grant DMS-1522593. 

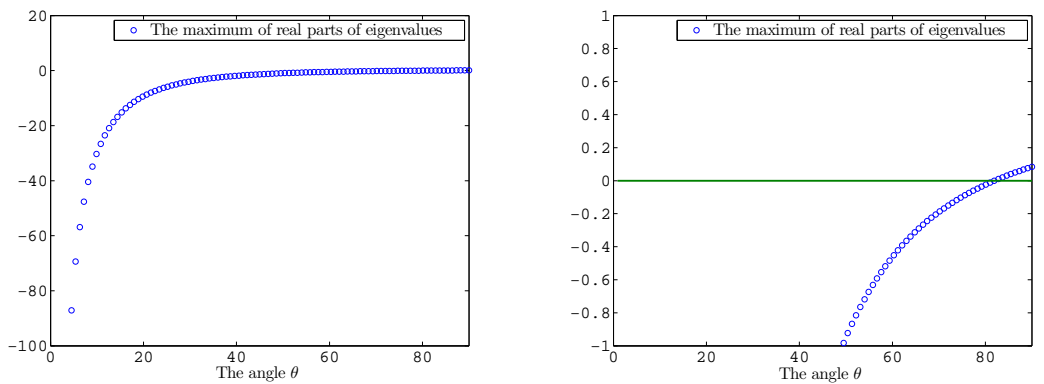

Figure B.12: The maximum of real parts in the eigenvalues of spatial discretization operator $\mathcal{L}_{h}$ in the $2 \mathrm{D}$ modified scheme with polynomials of degree 5 basis, defined on the triangle $A B C$ illustrated in Figure B.11 for solving (B.1).

\section{References}

[1] G. Strang, A. E. Berger, The change in solution due to change in domain, in: AMS Symposium on Partial Differential Equations, Berkeley, 1971, pp. 199-206.

[2] V. Thomée, Polygonal domain approximation in Dirichlet's problem, IMA Journal of Applied Mathematics 11 (1) (1973) 33-44. doi:10.1093/imamat/11.1.33.

[3] P. G. Ciarlet, The finite element method for elliptic problems, Elsevier, 1978.

[4] W. Gordon, C. Hall, Transfinite element methods: Blending-function interpolation over arbitrary curved element domains, Numerische Mathematik 21 (2) (1973) 109-129. doi:10.1007/BF01436298.

[5] F. Bassi, S. Rebay, High-order accurate discontinuous finite element solution of the 2D Euler equations, Journal of computational physics 138 (2) (1997) 251-285. doi:10.1006/jcph.1997.5454.

[6] J. S. Hesthaven, T. Warburton, Nodal high-order methods on unstructured grids: I. time-domain solution of Maxwell's equations, Journal of Computational Physics 181 (1) (2002) 186-221. doi:10.1006/jcph.2002.7118.

[7] J. Hesthaven, T. Warburton, Nodal Discontinuous Galerkin Methods: Algorithms, Analysis, and Applications, Texts in Applied Mathematics, Springer, 2007.

[8] D. A. Kopriva, Implementing spectral methods for partial differential equations: Algorithms for scientists and engineers, Springer Science \& Business Media, 2009. 
[9] I. Lomtev, R. Kirby, G. Karniadakis, A discontinuous Galerkin ALE method for compressible viscous flows in moving domains, Journal of Computational Physics 155 (1) (1999) 128-159. doi:10.1006/jcph.1999.6331.

[10] Z. Wang, H. Gao, A unifying lifting collocation penalty formulation including the discontinuous Galerkin, spectral volume/difference methods for conservation laws on mixed grids, Journal of Computational Physics 228 (21) (2009) 8161-8186. doi:0.1016/j.jcp.2009.07.036.

[11] T. Warburton, A low storage curvilinear discontinuous Galerkin timedomain method for electromagnetics, in: Electromagnetic Theory (EMTS), 2010 URSI International Symposium on, 2010, pp. 996-999. doi:10.1109/URSI-EMTS . 2010.5637392.

[12] T. Warburton, A low-storage curvilinear discontinuous Galerkin method for wave problems, SIAM Journal on Scientific Computing 35 (4) (2013) A1987-A2012. doi:10.1137/120899662.

[13] L. Krivodonova, M. Berger, High-order accurate implementation of solid wall boundary conditions in curved geometries, Journal of Computational Physics 211 (2) (2006) 492 - 512. doi:10.1016/j.jcp.2005.05.029.

[14] B. Cockburn, M. Solano, Solving convection-diffusion problems on curved domains by extensions from subdomains, Journal of Scientific Computing 59 (2) (2014) 512-543. doi:10.1007/s10915-013-9776-y.

[15] B. Cockburn, W. Qiu, M. Solano, A priori error analysis for HDG methods using extensions from subdomains to achieve boundary conformity, Mathematics of Computation 83 (286) (2014) 665-699. doi:10.1090/S0025-5718-2013-02747-0.

[16] S. Tan, C.-W. Shu, Inverse Lax-Wendroff procedure for numerical boundary conditions of conservation laws, Journal of Computational Physics 229 (21) (2010) 8144-8166. doi:10.1016/j.jcp.2010.07.014.

[17] S. Tan, C.-W. Shu, A high order moving boundary treatment for compressible inviscid flows, Journal of Computational Physics 230 (15) (2011) 6023-6036. doi:10.1016/j.jcp.2011.04.011.

[18] S. Tan, C.-W. Shu, Inverse Lax-Wendroff procedure for numerical boundary conditions of hyperbolic equations: Survey and new developments, in: Advances in Applied Mathematics, Modeling, and Computational Science, Springer, 2013, pp. 41-63. doi:10.1007/978-1-4614-5389-5_3.

[19] S. Tan, C. Wang, C.-W. Shu, J. Ning, Efficient implementation of high order inverse Lax-Wendroff boundary treatment for conservation laws, Journal of Computational Physics 231 (6) (2012) 2510-2527. doi:10.1016/j.jcp.2011.11.037. 
[20] X. Zhang, S. Tan, A simple and accurate discontinuous Galerkin scheme for modeling scalar-wave propagation in media with curved interfaces, Geophysics 80 (2) (2015) T83-T89. doi:10.1190/geo2014-0164.1.

[21] B. Cockburn, C.-W. Shu, The local discontinuous Galerkin method for time-dependent convection-diffusion systems, SIAM Journal on Numerical Analysis 35 (6) (1998) 2440-2463. doi:10.1137/S0036142997316712.

[22] C.-S. Chou, C.-W. Shu, Y. Xing, Optimal energy conserving local discontinuous Galerkin methods for second-order wave equation in heterogeneous media, Journal of Computational Physics 272 (2014) 88-107. doi:10.1016/j.jcp.2014.04.009.

[23] B. Cockburn, C.-W. Shu, TVB Runge-Kutta local projection discontinuous Galerkin finite element method for conservation laws. II. General framework, Mathematics of computation 52 (186) (1989) 411-435. doi:/10.1090/S0025-5718-1989-0983311-4.

[24] J. Qiu, C.-W. Shu, Runge-Kutta Discontinuous Galerkin Method Using WENO Limiters, SIAM Journal on Scientific Computing 26 (3) (2005) 907-929. doi:10.1137/S1064827503425298.

[25] X. Zhong, C.-W. Shu, A simple weighted essentially nonoscillatory limiter for Runge-Kutta discontinuous Galerkin methods, Journal of Computational Physics 232 (1) (2013) 397-415. doi:10.1016/j . jcp.2012.08.028.

[26] X. Zhang, C.-W. Shu, On maximum-principle-satisfying high order schemes for scalar conservation laws, Journal of Computational Physics 229 (9) (2010) 3091-3120. doi:10.1016/j.jcp.2009.12.030.

[27] X. Zhang, C.-W. Shu, On positivity-preserving high order discontinuous Galerkin schemes for compressible Euler equations on rectangular meshes, Journal of Computational Physics 229 (23) (2010) 8918-8934. doi:10.1016/j.jcp.2010.08.016.

[28] X. Zhang, Y. Xia, C.-W. Shu, Maximum-principle-satisfying and positivitypreserving high order discontinuous Galerkin schemes for conservation laws on triangular meshes, Journal of Scientific Computing 50 (1) (2012) 29-62. doi:10.1007/s10915-011-9472-8.

[29] A. Ditkowski, K. Dridi, J. S. Hesthaven, Convergent cartesian grid methods for Maxwell's equations in complex geometries, Journal of Computational Physics 170 (1) (2001) 39-80. doi:10.1006/jcph.2001.6719.

[30] H. Fahs, Development of a hp-like discontinuous Galerkin time-domain method on non-conforming simplicial meshes for electromagnetic wave propagation, International Journal of Numerical Analysis and Modeling 6 (2) (2009) 193-216. 
[31] C. Tam, J. Hardin, Second computational aeroacoustics workshop on benchmark problems, NASA, 1997.

[32] T. Toulorge, Y. Reymen, W. Desmet, A 2d discontinuous Galerkin method for aeroacoustics with curved boundary treatment, in: Proceedings of International Conference on Noise and Vibration Engineering (ISMA2008), Citeseer, 2008, pp. 565-578. 